\title{
FLUCTUATIONS IN RANDOM COMPLEX ZEROES: ASYMPTOTIC NORMALITY REVISITED
}

\author{
F. NAZAROV AND M. SODIN
}

Abstract. The Gaussian Entire Function

$$
F(z)=\sum_{k=0}^{\infty} \zeta_{k} \frac{z^{k}}{\sqrt{k !}}
$$

$\left(\zeta_{0}, \zeta_{1}, \ldots\right.$ are Gaussian i.i.d. complex random coefficients) is distinguished by the distribution invariance of its zero set with respect to the isometries of the complex plane. We find close to optimal conditions on a function $h$ that yield asymptotic normality of linear statistics of zeroes

$$
n(R, h)=\sum_{a: F(a)=0} h\left(\frac{a}{R}\right)
$$

when $R \rightarrow \infty$, and construct examples of functions $h$ with abnormal fluctuations of linear statistics. We show that the fluctuations of $n(R, h)$ are asymptotically normal when $h$ is either a $C_{0}^{\alpha}$-function with $\alpha>1$, or a $C_{0}^{\alpha}$-function with $\alpha \leqslant 1$ such that the variance of $n(R, h)$ is at least $R^{-2 \alpha+\varepsilon}$ with some $\varepsilon>0$.

These results complement our recent results from "Clustering of correlation functions for random complex zeroes", where, using different methods, we prove that the fluctuations of $n(R, h)$ are asymptotically normal when $h$ is bounded, and the variance of $n(R, h)$ grows at least as a positive power of $R$.

\section{Introduction AND MAIN RESUlts}

1.1. Consider the Gaussian entire function (G.E.F., for short)

$$
F(z)=\sum_{k=0}^{\infty} \zeta_{k} \frac{z^{k}}{\sqrt{k !}}
$$

where $\zeta_{n}$ are standard independent Gaussian complex variables (that is, the density of $\zeta_{n}$ on the complex plane is $\frac{1}{\pi} e^{-|\zeta|^{2}}$ ). A remarkable feature of the random zero set $\mathcal{Z}_{F}=F^{-1}\{0\}$ is its distribution invariance with respect to the isometries of the plane. The rotation invariance of $\mathcal{Z}_{F}$ is obvious since the distribution of $F$ is rotation invariant itself. Though the distribution of $F$ is not translation invariant at all, the translation invariance of the zero process $\mathcal{Z}_{F}$ follows from the fact that, for every $w \in \mathbb{C}$, the Gaussian processes $F(z+w)$ and $F_{w}(z)=F(z) e^{z \bar{w}+\frac{1}{2}|w|^{2}}$

Date: February 21, 2010.

F.N. is partially supported by the National Science Foundation, DMS grant 0501067. M.S. is partially supported by the Israel Science Foundation of the Israel Academy of Sciences and Humanities, grant 171/07. 
have the same distributions. The latter follows by inspection of the covariances: $\mathbb{E}\left\{F_{w}\left(z_{1}\right) \overline{F_{w}\left(z_{2}\right)}\right\}=e^{z_{1} \overline{z_{2}}+z_{1} \bar{w}+\overline{z_{2}} w+|w|^{2}}=e^{\left(z_{1}+w\right) \overline{\left(z_{2}+w\right)}}=\mathbb{E}\left\{F\left(z_{1}+w\right) \overline{F\left(z_{2}+w\right)}\right\}$.

By Calabi's rigidity [17, Section 3], [8, Section 2.5], $F(z)$ is essentially the only Gaussian functions analytic in $\mathbb{C}$ with the distribution of zeroes invariant with respect to the isometries of the plane. The random zero process $\mathcal{Z}_{F}$ together with similar models related to other one-dimensional complex geometries was introduced in the works of Bogomolny, Bohigas, Leboeuf [3], Hannay [6] and Kostlan [9]. After that the zero process $\mathcal{Z}_{F}$ has been studied from different points of view. A brief non-technical introduction can be found in [12].

1.2. Linear statistics of zeroes and their variances. One of the ways to understand the asymptotic behaviour of the zero process $\mathcal{Z}_{F}$ is to introduce the random variable

$$
n(R, h)=\sum_{a \in \mathcal{Z}_{F}} h\left(\frac{a}{R}\right)
$$

called the linear statistics of $\mathcal{Z}_{F}$ and to study its behavior as $R \rightarrow \infty$. Here, $h$ is a function that we always assume real-valued, not identically zero, and belonging to $\left(L^{1} \cap L^{2}\right)\left(\mathbb{R}^{2}\right)$. Since $\mathcal{Z}_{F}$ has a translation-invariant distribution, we have

$$
\mathbb{E}\{n(R, h)\}=C R^{2} \int_{\mathbb{R}^{2}} h .
$$

A straightforward computation shows that $C=\frac{1}{\pi}$ (see, for instance, [18, Part I]).

Denote by

$$
\mathbb{V}(R, h)=\mathbb{E}\{n(R, h)-\mathbb{E} n(R, h)\}^{2}
$$

the variance of $n(R, h)$, and by $\sigma(R, h)=\sqrt{\mathbb{V}(R, h)}$ its standard deviation. In [4], Forrester and Honner found that if $h \in C_{0}^{2}$ (i.e., $h$ is a $C^{2}$-function with compact support), then

$$
\mathbb{V}(R, h)=\frac{\zeta(3)+o(1)}{16 \pi R^{2}}\|\Delta h\|_{L^{2}}^{2}, \quad R \rightarrow \infty,
$$

while for $h=\mathbb{1}_{G}$ (the indicator function of a bounded domain $G$ with piecewise smooth boundary)

$$
\mathbb{V}\left(R, \mathbb{1}_{G}\right)=\frac{\zeta(3 / 2)+o(1)}{8 \pi^{3 / 2}} R L(\partial G), \quad R \rightarrow \infty .
$$

Here, $\zeta(\cdot)$ is Riemann's zeta-function. Later, Sodin and Tsirelson [18, Part I] and Shiffman and Zelditch [16] found different approaches to asymptotic computation of the variance, which work in more general contexts. Our first result is an exact formula for the variance $\mathbb{V}(R, h)$ valid for arbitrary functions $h \in\left(L^{1} \cap L^{2}\right)\left(\mathbb{R}^{2}\right)$.

In what follows, $X \lesssim Y$ means that $X \leqslant C \cdot Y$ with a positive numerical constant $C$, and $X \simeq Y$ means that $X \lesssim Y$ and $Y \lesssim X$ simultaneously. By

$$
\widehat{h}(\lambda)=\int_{\mathbb{R}^{2}} h(x) e^{-2 \pi \mathrm{i}\langle\lambda, x\rangle} \mathrm{d} A(x)
$$

we denote the Fourier transform of $h$. 
Theorem 1.1. For every non-zero function $h \in\left(L^{1} \cap L^{2}\right)\left(\mathbb{R}^{2}\right)$ and each $R>0$, one has

$$
\mathbb{V}(R, h)=R^{2} \int_{\mathbb{R}^{2}}|\widehat{h}(\lambda)|^{2} M\left(R^{-1} \lambda\right) \mathrm{d} A(\lambda)
$$

where

$$
M(\lambda)=\pi^{3}|\lambda|^{4} \sum_{\alpha \geqslant 1} \frac{1}{\alpha^{3}} e^{-\frac{\pi^{2}}{\alpha}|\lambda|^{2}} .
$$

Noticing that $M(\lambda) \simeq \min \left(|\lambda|^{4}, 1\right)$, we get

$$
\mathbb{V}(R, h) \simeq R^{-2} \int_{|\lambda| \leqslant R}|\widehat{h}(\lambda)|^{2}|\lambda|^{4} \mathrm{~d} A(\lambda)+R^{2} \int_{|\lambda| \geqslant R}|\widehat{h}(\lambda)|^{2} \mathrm{~d} A(\lambda) .
$$

Observe that the right-hand side of (11) interpolates the $L^{2}$-norm of the function $h$ and the $L^{2}$-norm of its Laplacian $\Delta h$.

1.3. Normality of fluctuations. The question about the asymptotic normality of fluctuations of $n(R, h)$ is more delicate. For compactly supported $C^{2}$-functions $h$, the asymptotic normality was proven by Sodin and Tsirelson in [18, Part I]. The proof was based on the method of moments, and the moments were computed using the diagram technique 1 . After some modification, the argument from [18, Part I] also works in the case when $h=\mathbb{1}_{G}$ where $G$ is a bounded domain with piecewise smooth boundary. Krishnapur noticed in his PhD Thesis [10] that the same argument also works when $h=\mathbb{1}_{G} * \mathbb{1}_{G}$ (the convolution square). In the latter case, the variance tends to a constant. In a recent work [20], Tsirelson found the asymptotics of the logarithm of the characteristic functional $\mathbb{E} e^{\lambda n(R, h)}$, where $h$ is a compactly supported $C^{2}$-function, when $R \rightarrow \infty, \lambda \rightarrow 0$ in such way that $\lambda \log ^{2} R \rightarrow 0$. Among other things, this gives a different proof of asymptotic normality of smooth linear statistics of random complex zeroes.

Let $C_{0}^{\alpha}$ be the class of compactly supported $C^{\alpha}$-functions. In this paper, we explore what happens for $C_{0}^{\alpha}$-functions with $0<\alpha<2$. We say that the linear statistics $n(R, h)$ have asymptotically normal fluctuations if the normalized linear statistics

$$
\frac{n(R, h)-\mathbb{E} n(R, h)}{\sigma(R, h)}
$$

converge in distribution to the standard (real) Gaussian random variable as $R \rightarrow$ $\infty$.

Theorem 1.2. Suppose that $h \in C_{0}^{\alpha}$ with some $\alpha>0$, and that for some $\varepsilon>0$ and each sufficiently big $R$, we have

$$
\sigma(R, h)>R^{-\alpha+\varepsilon} .
$$

Then the linear statistics $n(R, h)$ have asymptotically normal fluctuations.

\footnotetext{
${ }^{1}$ Note that using the variance estimate (1), one readily extends this result to the functions $h$ in the Sobolev space $W_{2}^{2}$; i.e., to the functions $h$ such that

$$
\int_{\mathbb{R}^{2}}|\widehat{h}(\lambda)|^{2}\left(1+|\lambda|^{4}\right) \mathrm{d} A(\lambda)<\infty .
$$
}


Note that by (11), we always have $\sigma(R, h) \gtrsim c(h) R^{-1}$ with positive $c(h)$ independent of $R$. Hence, for $\alpha>1$, condition (2) holds automatically.

Corollary 1.3. Suppose that $h \in C_{0}^{\alpha}$ with $\alpha>1$. Then the linear statistics $n(R, h)$ have asymptotically normal fluctuations.

We mention that there are plenty of nice $C_{0}^{\alpha}$-functions with $\alpha \leqslant 1$ satisfying condition (2) of Theorem 1.2, like, say, $h(x)=(1-|x|)_{+}^{\alpha}$ for which $\sigma(R, h) \simeq R^{\frac{1}{2}-\alpha}$.

1.4. Few questions. We do not know whether asymptotic normality holds for all functions $h \in C_{0}^{1}$, or whether the condition $R^{\alpha} \sigma(R, h) \rightarrow \infty$ is already sufficient for asymptotic normality of linear statistics associated with a $C_{0}^{\alpha}$-function. Also, we believe that the assertion of Theorem 1.2 can be extended to functions $h \in C^{\alpha} \cap L_{0}^{2}$ with $-1<\alpha<0$ but our current techniques seem insufficient to handle this case properly.

1.5. Bounded test-functions. It is worth mentioning that Theorem 1.2 is complemented by our recent results [11] where, using different methods, we prove that the fluctuations of $n(R, h)$ are asymptotically normal when $h$ is bounded, and the variance of $n(R, h)$ grows at least as a positive power of $R$.

1.6. Test-functions with abnormal fluctuations in linear statistics. We will see that for every $\alpha \in(0,1)$, the function $h=|x|^{\alpha} \psi(x)$, where $\psi$ is a smooth cut-off that equals 1 in a neighborhood of the origin, yields an abnormal behavior of the corresponding linear statistics. Clearly, $h \in C_{0}^{\alpha}$ and it is possible to show that $\sigma(R, h) \simeq R^{-\alpha}$. This shows that Theorem 1.2 is sharp on a rough power scale.

The reason for the loss of asymptotic normality is that only a small neighbourhood of the origin containing a bounded number of zeroes of $F$ contributes to the variance of $n(R, h)$, which is not consistent with the idea of normal fluctuations of linear statistics.

1.7. Comparing random complex zeroes with limiting eigenvalue process for the Ginibre ensemble. It is interesting to juxtapose our results with what is known for the $N \rightarrow \infty$ limit of the eigenvalue point process $\mathcal{G}$ of the Ginibre ensemble of $N \times N$ random matrices with independent standard Gaussian complex entries. The process $\mathcal{G}$ is a determinantal one, with the kernel $K(z, w)=e^{z \bar{w}}$ (curiously, this is the same kernel that appears as the covariance for the G.E.F. $F(z))$. It is known that in this case the variance of linear statistics never decays, for $W_{1}^{2}$-functions $h$ it tends to the limit proportional to $\|\nabla h\|_{L^{2}}$, and it never grows faster than $R^{2}$. A counterpart of our result proven in 11] is a theorem of Soshnikov. In [19], he proved among other things that for arbitrary determinantal point processes, the fluctuations of linear statistics associated with a compactly supported bounded positive function are normal if the variance grows at least as a positive power of expectation as the intensity tends to infinity. A counterpart of the limiting case $\alpha=2$ in Theorem 1.2 (that is, of the result from [18, Part I]) was recently found by Rider and Virág in [15. They proved that the fluctuations for linear statistics of process $\mathcal{G}$ are normal when the test function $h$ belongs to 
the Sobolev space $W_{1}^{2}$. It is not clear whether there is any meaningful statement interpolating the theorems of Soshnikov and Rider and Virág. It can happen that our Theorem 1.2 simply has no interesting counterpart for the process $\mathcal{G}$. It is also worth mentioning that the proofs in the determinantal case are quite different from ours. They are based on peculiar combinatorial identities for the cumulants of linear statistics that are a special feature of determinantal point processes.

1.8. Idea of the proof of Theorem 1.2, To prove Theorem 1.2, we follow a classical idea of S. N. Bernstein [1] and approximate the random variable $n(R, h)$ by a sum of a large number of independent random variables with negligible error 2 . It is worth mentioning that such approximation becomes possible only after we separate the high and the low frequencies in $h$. In this approach, independence appears as a consequence of the almost independence property of G.E.F.'s found in [13, 14. Roughly speaking, it says that if the compact sets $K_{j} \subset \mathbb{R}^{2}$ are well-separated from each other, then the restrictions of the normalized processes $F^{*}(z)=F(z) e^{-\frac{1}{2}|z|^{2}}$ to $K_{j}$ can be simultaneously approximated by the restrictions of normalized independent realizations of G.E.F.'s with very high probability.

Acknowledgments. We are grateful to Yuri Makarychev who suggested the idea of the proof of Lemma 3.2 which is a central part in our proof of the asymptotic independence theorem 3.1, and to Manjunath Krishnapur and Boris Tsirelson for very helpful discussions.

\section{THE VARIANCE}

2.1. Proof of Theorem 1.1. We start with several lemmas. First, we find the coefficients of the Hermite-Ito orthogonal expansion of the function $\log |\zeta|$ in the Hilbert space $\mathcal{H}=L^{2}\left(\mathbb{C}, \frac{1}{\pi} e^{-|\zeta|^{2}}\right)$. We use the notation traditional in the probability theory.

Denote by $\mathcal{P}_{m}$ the linear subspace of $\mathcal{H}$ consisting of algebraic polynomials in $z, \bar{z}$ of degree at most $m$, and set $\mathcal{H}^{: m:}=\mathcal{P}_{m} \ominus P_{m-1}$. By $: \zeta^{\alpha} \bar{\zeta}^{\beta}:\left(\alpha, \beta \in \mathbb{Z}_{+}\right)$we denote the orthogonal basis in $\mathcal{H}$ obtained by projecting the polynomials $\zeta^{\alpha} \bar{\zeta}^{\beta}$ to $\mathcal{H}^{: m:}, m=\alpha+\beta$. A short computation shows that $\left\|: \zeta^{\alpha} \bar{\zeta}^{\beta}:\right\|^{2}=\alpha ! \beta !$ [5. Example 3.32]. Since $\log |\zeta|$ is a radial function, its expansion in this basis contains only the radial polynomials; i.e., the ones with $\alpha=\beta$. The coefficients of this expansion can be readily computed:

Lemma 2.1. We have

$$
\log |\zeta|=c_{0}+\sum_{\alpha \geqslant 1} \frac{c_{2 \alpha}}{\alpha !}:|\zeta|^{2 \alpha}
$$

with $c_{0}=\mathbb{E}\{\log |\zeta|\}=-\frac{1}{2} \gamma$ ( $\gamma$ is the Euler constant) and $c_{2 \alpha}=(-1)^{\alpha+1} \frac{1}{2 \alpha}$ for $\alpha \geqslant 1$.

\footnotetext{
${ }^{2}$ The surgery technique developed in Tsirelson's paper [20] is also reminiscent of Bernstein's idea.
} 
Proof of Lemma 2.1: Suppose $\alpha \geqslant 1$. Let $\mathcal{H}_{\text {rad }}$ be the subspace of $\mathcal{H}$ that consists of radial functions. If $\Phi \in \mathcal{H}_{\mathrm{rad}}$, then letting $\Phi(\zeta)=\varphi\left(|\zeta|^{2}\right)$, we get

$$
\|\Phi\|_{\mathcal{H}}^{2}=\frac{1}{\pi} \int_{0}^{2 \pi} \mathrm{d} \theta \int_{0}^{\infty}\left|\varphi\left(r^{2}\right)\right|^{2} e^{-r^{2}} r \mathrm{~d} r=\int_{0}^{\infty}|\varphi(t)|^{2} e^{-t} \mathrm{~d} t .
$$

This identifies the subspace $\mathcal{H}_{\text {rad }}$ with the space $L^{2}\left(\mathbb{R}_{+}, e^{-t}\right)$. Therefore, $\frac{1}{\alpha !}:|\zeta|^{2 \alpha}$ : $=(-1)^{\alpha} L_{\alpha}\left(|\zeta|^{2}\right)$, where

$$
L_{\alpha}(t)=\frac{1}{\alpha !} e^{t} \frac{\mathrm{d}^{\alpha}}{\mathrm{d} t^{\alpha}}\left(t^{\alpha} e^{-t}\right)
$$

are Laguerre orthogonal polynomials. In our case, $\varphi(t)=\frac{1}{2} \log |t|$, whence

$$
\begin{aligned}
c_{2 \alpha}=\frac{(-1)^{\alpha}}{2 \alpha !} \int_{0}^{\infty} \log t\left(t^{\alpha} e^{-t}\right)^{(\alpha)} \mathrm{d} t= & -\frac{(-1)^{\alpha}}{2 \alpha !} \int_{0}^{\infty} \frac{1}{t}\left(t^{\alpha} e^{-t}\right)^{(\alpha-1)} \mathrm{d} t \\
& =\ldots=-\frac{(-1)^{\alpha}}{2 \alpha} \int_{0}^{\infty} e^{-t} \mathrm{~d} t=\frac{(-1)^{\alpha+1}}{2 \alpha}
\end{aligned}
$$

for all $\alpha \geqslant 1$. For $\alpha=0$, we have

$$
c_{0}=\mathbb{E}\{\log |\zeta|\}=\frac{1}{2} \int_{0}^{\infty}(\log t) e^{-t} \mathrm{~d} t=\frac{1}{2} \Gamma^{\prime}(1)=-\frac{1}{2} \gamma .
$$

Hence the lemma.

Lemma 2.2. Suppose $\zeta_{i}, i=1,2$, are standard complex Gaussian random variables with $\left|\mathbb{E}\left\{\zeta_{1} \overline{\zeta_{2}}\right\}\right|=\rho$. Then

$$
\mathbb{E}\left(\log \left|\zeta_{1}\right|-c_{0}\right)\left(\log \left|\zeta_{2}\right|-c_{0}\right)=\frac{1}{4} \sum_{\alpha \geqslant 1} \frac{\rho^{2 \alpha}}{\alpha^{2}},
$$

where $c_{0}=\mathbb{E} \log \left|\zeta_{i}\right|=-\frac{1}{2} \gamma$.

Proof of Lemma 2.2: Using that

$$
\mathbb{E}\left(\frac{1}{\alpha !}:\left|\zeta_{1}\right|^{2 \alpha}:\right)\left(\frac{1}{\beta !}:\left|\zeta_{2}\right|^{2 \beta}:\right)= \begin{cases}\rho^{2 \alpha} & \text { if } \alpha=\beta \\ 0 & \text { otherwise }\end{cases}
$$

[5. Theorem 3.9], we readily derive the result from Lemma 2.1.

We note that a different proof of this lemma can be found in [16, Lemma 3.3].

Now, let $f$ be an arbitrary Gaussian analytic function in a domain $G \subset \mathbb{C}$, and let $f^{*}(x)=\frac{f(x)}{\sqrt{\mathbb{E}|f(x)|^{2}}}$. Denote by $n_{f}=\sum_{a: f(a)=0} \delta_{a}$ the (random) counting measure of its zeroes, and set $\bar{n}_{f}=n_{f}-\mathbb{E} n_{f}$. Define the normalized 2-point correlation measure $\nu$ of zeroes of $f$ on $G \times G$ by $\nu=\mathbb{E}\left\{\bar{n}_{f} \times \bar{n}_{f}\right\}$. Knowing the two-point measure $\nu$, we easily recover the variance of the random variable $n(R, h)$ :

$$
\mathbb{V}(R, h)=\iint_{\mathbb{R}^{2} \times \mathbb{R}^{2}} h\left(\frac{x_{1}}{R}\right) h\left(\frac{x_{2}}{R}\right) \mathrm{d} \nu\left(x_{1}, x_{2}\right) .
$$


Lemma 2.3.

$$
\nu=\frac{1}{16 \pi^{2}} \sum_{\alpha \geqslant 1} \frac{1}{\alpha^{2}} \Delta_{x_{1}} \Delta_{x_{2}}\left(\rho^{2 \alpha}\right) \quad \text { (as a distribution), }
$$

where $\rho\left(x_{1}, x_{2}\right)=\left|\mathbb{E}\left(f^{*}\left(x_{1}\right) \overline{f^{*}\left(x_{2}\right)}\right)\right|$ is the normalized correlation coefficient of complex Gaussian random variables $f\left(x_{1}\right)$ and $f\left(x_{2}\right)$, and $\Delta_{x_{j}}$ are (distributional) Laplacians acting on the variables $x_{j}, j=1,2$.

Proof of Lemma 2.3: We have $\bar{n}_{f}=\frac{1}{2 \pi} \Delta \log \left|f^{*}\right|$ (this is the Edelman-Kostlan formula, see [8, 17]), whence

$$
\nu=\left(\frac{1}{2 \pi}\right)^{2} \Delta_{x_{1}} \Delta_{x_{2}} \mathbb{E}\left\{\log \left|f^{*}\left(x_{1}\right)\right| \log \left|f\left(x_{2}^{*}\right)\right|\right\} .
$$

Lemma 2.2 with $\zeta_{i}=f^{*}\left(x_{j}\right), j=1,2$, yields (up to terms constant in $x_{1}$ or $x_{2}$ that vanish after we apply the Laplacians in $x_{1}$ and $x_{2}$ )

$$
\mathbb{E}\left\{\log \left|f^{*}\left(x_{1}\right)\right| \log \left|f\left(x_{2}^{*}\right)\right|\right\}=\frac{1}{4} \sum_{\alpha \geqslant 1} \frac{1}{\alpha^{2}} \rho\left(x_{1}, x_{2}\right)^{2 \alpha}+\langle\text { negligible terms }\rangle,
$$

which implies Lemma 2.3.

Proof of Theorem 1.1: It suffices to prove the theorem for $R=1$. The rest readily follows by scaling

$$
x \mapsto R x, \quad h \mapsto h\left(R^{-1} \cdot\right), \quad \lambda \mapsto R^{-1} \lambda, \quad \widehat{h} \mapsto R^{2} \widehat{h}(R \cdot) .
$$

We have

$$
\mathbb{V}(1, h)=\iint_{\mathbb{R}^{2} \times \mathbb{R}^{2}} h\left(x_{1}\right) h\left(x_{2}\right) \mathrm{d} \nu\left(x_{1}, x_{2}\right) .
$$

To compute the 2-point measure $\nu$, we use Lemma 2.2. The normalized correlation coefficient $\rho\left(x_{1}, x_{2}\right)$ of the G.E.F. $F$ equals

$$
\rho\left(x_{1}, x_{2}\right)=\exp \left[-\operatorname{Re}\left(x_{1} \bar{x}_{2}\right)-\frac{1}{2}\left|x_{1}\right|^{2}-\frac{1}{2}\left|x_{2}\right|^{2}\right]=\exp \left[-\frac{1}{2}\left|x_{1}-x_{2}\right|^{2}\right],
$$

whence,

$$
\mathbb{V}(1, h)=\frac{1}{16 \pi^{2}} \sum_{\alpha \geqslant 1} \frac{1}{\alpha^{2}} \iint_{\mathbb{R}^{2} \times \mathbb{R}^{2}} h\left(x_{1}\right) h\left(x_{2}\right) \Delta_{x_{1}} \Delta_{x_{2}} e^{-\alpha\left|x_{1}-x_{2}\right|^{2}} \mathrm{~d} A\left(x_{1}\right) \mathrm{d} A\left(x_{2}\right) .
$$

Starting from here, we shall assume that $h \in C_{0}^{2}$. It doesn't really matter because it is possible to show that both sides of the last equation are continuous functionals in $\left(L^{1} \cap L^{2}\right)\left(\mathbb{R}^{2}\right)$.

Now, we use the identity

$$
\iint_{\mathbb{R}^{2} \times \mathbb{R}^{2}} K\left(x_{1}-x_{2}\right) g\left(x_{1}\right) g\left(x_{2}\right) \mathrm{d} A\left(x_{1}\right) \mathrm{d} A\left(x_{2}\right)=\int_{\mathbb{R}^{2}} \widehat{K}(\lambda)|\widehat{g}(\lambda)|^{2} \mathrm{~d} A(\lambda),
$$

with $g(x)=\Delta h(x)$ and $K(x)=e^{-\alpha|x|^{2}}$. With our normalization of the Fourier transform we have

$$
\widehat{g}(\lambda)=-4 \pi^{2}|\lambda|^{2} \widehat{h}(\lambda), \quad \widehat{K}(\lambda)=\frac{\pi}{\alpha} e^{-\pi^{2}|\lambda|^{2} / \alpha} .
$$


Finally, we get

with

$$
\mathbb{V}(1, h)=\int_{\mathbb{R}^{2}}|\widehat{h}(\lambda)|^{2} M(\lambda) \mathrm{d} A(\lambda)
$$

$$
M(\lambda)=\pi^{3}|\lambda|^{4} \sum_{\alpha \geqslant 1} \frac{1}{\alpha^{3}} e^{-\frac{\pi^{2}}{\alpha}|\lambda|^{2}}
$$

completing the proof of Theorem 1.1 .

Remark 2.4. Later, we will need the following observation, which can be proven in a similar way. Denote by $U$ the function $U(x)=\log |F(x)|-\frac{1}{2}|x|^{2}$. Let $g$ be an $L^{2}\left(\mathbb{R}^{2}\right)$-function with compact support. Then the variance of the random variable $\int_{\mathbb{R}^{2}} g U \mathrm{~d} A$ is $\lesssim\|g\|_{L^{2}}^{2}$.

2.2. Corollaries. Later, we will need the following three corollaries to Theorem 1.1 .

Corollary 2.5. For each $h \in\left(L^{1} \cap L^{2}\right)\left(\mathbb{R}^{2}\right)$, we have

$$
\mathbb{V}(R, h) \lesssim R^{2}\|h\|_{L^{2}}^{2}, \quad 0<R<\infty,
$$

and

$$
\mathbb{V}(R, h)=o\left(R^{2}\right), \quad R \rightarrow \infty
$$

By $W_{2}^{2}$ we denote the Sobolev space of $L^{2}$-functions $h$ with $\Delta h \in L^{2}$.

Corollary 2.6. For each $h \in L^{1} \cap W_{2}^{2}$,

$$
\mathbb{V}(R, h) \lesssim R^{-2}\|\Delta h\|_{L^{2}}^{2}, \quad 0<R<\infty .
$$

Corollary 2.7. For any cut-off function $\chi$ with $|\widehat{\chi}(\lambda)| \lesssim\left(1+|\lambda|^{2}\right)^{-1}$, we have

$$
\mathbb{V}(R, h) \gtrsim R^{-2}\left\|\Delta\left(h * \chi_{R}\right)\right\|_{L^{2}}^{2}, \quad 0<R<\infty .
$$

Here, $\chi_{R}=R^{2} \chi(R \cdot)$.

\section{Almost independence}

The proof of Theorem 1.2 is based on the almost independence property of G.E.F. introduced in [13, 14]. It says that if $\left\{K_{j}\right\}$ is a collection of well-separated compact sets in $\mathbb{C}$, then the restrictions of normalized processes $\left.F^{*}\right|_{K_{j}}$ can be simultaneously approximated by restrictions $\left.F_{j}^{*}\right|_{K_{j}}$ of normalized independent realizations of G.E.F.'s with very high probability. Here, and everywhere below, $F^{*}(z)=e^{-\frac{1}{2}|z|^{2}} F(z)$. In [13, 14] we used this idea in the case when $K_{j}$ were disks. The proofs given in those papers used Taylor expansions of the shifted G.E.F.'s $F\left(z+w_{j}\right) e^{-z \bar{w}_{j}} e^{-\frac{1}{2}\left|w_{j}\right|^{2}}$ where $w_{j}$ were the centers of the disks $K_{j}$. These proofs cannot be immediately extended to arbitrary compact sets $K_{j}$. Here, we give a more general version of this principle that does not assume anything about the structure of the sets $K_{j}$. 
Theorem 3.1. Let $F$ be a G.E.F. There exists a numerical constant $A>1$ with the following property. Given a family of compact sets $K_{j}$ in $\mathbb{C}$ with diameters $d\left(K_{j}\right)$, let $\rho_{j} \geqslant \sqrt{\log \left(3+d\left(K_{j}\right)\right)}$. Suppose that $A \rho_{j}$-neighbourhoods of the sets $K_{j}$ are pairwise disjoint. Then

$$
F^{*}=F_{j}^{*}+G_{j}^{*} \quad \text { on } K_{j}
$$

where $F_{j}$ are independent G.E.F.'s and

$$
\mathbb{P}\left\{\max _{K_{j}}\left|G_{j}^{*}\right| \geqslant e^{-\rho_{j}^{2}}\right\} \lesssim \exp \left[-e^{\rho_{j}^{2}}\right] .
$$

When proving this theorem, it will be convenient to treat complex Gaussian random variables as elements in a "big" Gaussian Hilbert space $\mathcal{H}$. First, we consider the Gaussian Hilbert space $\mathcal{H}_{F}$, which is a closure of finite linear combinations $U=\sum_{k} c_{k} F\left(z_{k}\right)$ with respect to the scalar product generated by the covariance: $\langle U, V\rangle=\mathbb{E}\{U \bar{V}\}$. We assume that the big space $\mathcal{H}$ consists of complex valued Gaussian random variables and contains countably many mutually orthogonal copies of $\mathcal{H}_{F}$. This will allow us to introduce new independent copies of some Gaussian random variables when necessary.

The proof of Theorem 3.1 goes as follows. First, for each compact set $K_{j}$, we choose a sufficiently dense net $Z_{j}$ and consider the bunch $N_{j}=\left\{v_{z}: z \in Z_{j}\right\}$ of unit vectors $v_{z}=F^{*}(z)$. Since the compact sets $K_{j}$ are well-separated, the bunches $N_{j}$ are almost orthogonal to each other. Then we slightly perturb the vectors $v_{z}$ without changing the angles between the vectors within each bunch $N_{j}$, making the bunches orthogonal to each other. More accurately, we construct new bunches $\widetilde{N}_{j}=\left\{\widetilde{v}_{z}: z \in Z_{j}\right\}$ so that for $z \in Z_{j}, \zeta \in Z_{k}$,

$$
\left\langle\widetilde{v}_{z}, \widetilde{v}_{\zeta}\right\rangle= \begin{cases}\left\langle v_{z}, v_{\zeta}\right\rangle & \text { for } j=k, \\ 0 & \text { for } j \neq k\end{cases}
$$

with good control of the errors $\left\|v_{z}-\widetilde{v}_{z}\right\|$. Then we extend the Gaussian bunches $\left\{\widetilde{v}_{z} e^{\frac{1}{2}|z|^{2}}: z \in Z_{j}\right\}$ to independent G.E.F.'s $F_{j}$. The difference $G_{j}=F-F_{j}$ is a random entire function that is small on the net $Z_{j}$ with probability very close to one. At the last step of the proof, we show that $G_{j}^{*}$ is small everywhere on $K_{j}$.

Proof of Theorem [3.1: We start with the construction of the net $Z_{j}$. We fix a unit lattice in $\mathbb{R}^{2}$ and denote by $\mathcal{C}_{j}$ a finite collection of the lattice points that is contained in a $\frac{1}{\sqrt{2}}$-neighbourhood of the compact set $K_{j}$ and is a $\frac{1}{\sqrt{2}}$-net for $K_{j}$. Then we consider the collection $\mathcal{D}_{j}$ of the unit disks centered at $\mathcal{C}_{j}$ and choose about $A^{2} \rho_{j}^{2}$ equidistant points on the boundary of each of these unit disks where $A$ is a sufficiently big constant to be chosen later. The collection of all these points will be our net $Z_{j}$. Then, for $z \in Z_{j}$, we introduce the bunch $N_{j}=\left\{v_{z}: z \in Z_{j}\right\} \subset \mathcal{H}$ of unit vectors $v_{z}=F^{*}(z)$. Since

$$
\left|\left\langle v_{z}, v_{\zeta}\right\rangle\right| \leqslant e^{-|z-\zeta|^{2} / 2}
$$

and since the compact sets $K_{j}$ are well separated, the vectors in the bunches $N_{j}$ and $N_{k}$ are almost orthogonal when $j \neq k$. 
Now, we replace almost orthogonal bunches by the orthogonal ones.

Lemma 3.2. There exist vectors $w_{z} \in \mathcal{H}$ such that

$$
\left\langle w_{z}, w_{\zeta}\right\rangle= \begin{cases}-\left\langle v_{z}, v_{\zeta}\right\rangle, & z \in Z_{j}, \zeta \in Z_{k}, j \neq k, \\ 0, & z, \zeta \in Z_{j}, \quad z \neq \zeta \\ e^{-\frac{1}{5} A^{2} \rho_{j}^{2}}, & z=\zeta,\end{cases}
$$

and

$$
\left\langle w_{z}, v_{\zeta}\right\rangle=0 \quad \text { for each } z, \zeta .
$$

Proof of Lemma 3.2: Consider the Hermitian matrix $\Gamma$ with the elements $\gamma(z, \zeta)$ defined by the right-hand side of (4). We will check that the matrix $\Gamma$ is positive definite. This will ensure the existence of vectors $w_{z}$ in $\mathcal{H} \ominus \operatorname{span}\left(v_{z}\right)$ with the Gram matrix $\Gamma$.

By the classical Gershgorin theorem, each eigenvalue of the matrix $\Gamma$ lies in one of the intervals

$$
(\gamma(z, z)-t(z), \gamma(z, z)+t(z)) \quad \text { with } \quad t(z)=\sum_{\zeta: \zeta \neq z}|\gamma(z, \zeta)| .
$$

Below, we check that for each $z, t(z)<\gamma(z, z)$; i.e, all eigenvalues of the matrix $\Gamma$ are positive, whence $\Gamma>0$.

Lemma 3.3. Let $z \in Z_{j}$. Then

$$
\sum_{k: k \neq j} \sum_{\zeta \in Z_{k}}\left|\left\langle v_{z}, v_{\zeta}\right\rangle\right|<e^{-\frac{1}{5} A^{2} \rho_{j}^{2}}
$$

provided that the constant $A$ in the assumptions of Theorem 3.1 is big enough.

Proof of Lemma 3.3: Fix $k \neq j$ and $c \in \mathcal{C}_{k}$. Denote by $D$ the unit disk centered at $c$. Then for each point $\zeta \in \partial D$, we have $|z-\zeta| \geqslant|z-c|-1$, and by (3)

$$
\left|\left\langle v_{z}, v_{\zeta}\right\rangle\right| \leqslant e^{-\frac{1}{4}|z-c|^{2}+1}
$$

whence

$$
\sum_{\zeta \in \partial D_{w}}\left|\left\langle v_{z}, v_{\zeta}\right\rangle\right| \leqslant A^{2} \rho_{k}^{2} e \cdot e^{-\frac{1}{4}|z-c|^{2}}
$$

and

$$
\sum_{\zeta \in Z_{k}}\left|\left\langle v_{z}, v_{\zeta}\right\rangle\right| \leqslant A^{2} e \sum_{c \in \mathcal{C}_{k}} \rho_{k}^{2} e^{-\frac{1}{4}|z-c|^{2}}
$$

Since the $A \rho_{j}$-neighbourhood of $K_{j}$ and the $A \rho_{k}$-neighbourhood of $K_{k}$ are disjoint, for each $z \in Z_{j}$ and $c \in \mathcal{C}_{k}$ with $j \neq k$, we have

$$
\rho_{k}<\rho_{j}+\rho_{k} \leqslant \frac{1}{A}\left(|z-c|+\operatorname{dist}\left(z, K_{j}\right)+\operatorname{dist}\left(c, K_{k}\right)\right) \leqslant \frac{1}{A}(|z-c|+\sqrt{2}+1) .
$$

Since $\rho_{k} \geqslant 1$ and $A$ is sufficiently big, we conclude that $\rho_{k}<\frac{2}{A}|z-c|$. Then

$$
\sum_{\zeta \in Z_{k}}\left|\left\langle v_{z}, v_{\zeta}\right\rangle\right| \leqslant 4 e \sum_{c \in \mathcal{C}_{k}}|z-c|^{2} e^{-\frac{1}{4}|z-c|^{2}}
$$


and

$$
\sum_{k: k \neq j} \sum_{\zeta \in Z_{k}}\left|\left\langle v_{z}, v_{\zeta}\right\rangle\right| \leqslant 4 e \sum_{k \neq j} \sum_{c \in \mathcal{C}_{k}}|z-c|^{2} e^{-\frac{1}{4}|z-c|^{2}} .
$$

To estimate the right-hand side, we introduce the counting measure $\mu$ of the set $\bigcup_{k: k \neq j} \mathcal{C}_{k}$. By our construction, for $z \in Z_{j}$, we have

$$
\mu(D(z, t)) \leqslant \begin{cases}C_{1} t^{2}, & \text { for } t \geqslant A \rho_{j}-C_{2}, \\ 0, & \text { for } t<A \rho_{j}-C_{2},\end{cases}
$$

with positive numerical constants $C_{1}$ and $C_{2}$. Then

$$
\begin{aligned}
4 e \sum_{k \neq j} \sum_{c \in \mathcal{C}_{k}}|z-c|^{2} e^{-\frac{1}{4}|z-c|^{2} / 2}=4 e \int_{\mathbb{R}^{2}}|z-c|^{2} e^{-\frac{1}{4}|z-c|^{2}} \mathrm{~d} \mu(c) & \\
=4 e \int_{0}^{\infty}\left(-2 t+\frac{1}{2} t^{3}\right) e^{-t^{2} / 4} \mu(D(z, t)) \mathrm{d} t & \leqslant C_{3} \int_{A \rho_{j}-C_{2}}^{\infty} t^{5} e^{-t^{2} / 4} \mathrm{~d} t<e^{-\frac{1}{5} A^{2} \rho_{j}^{2}}
\end{aligned}
$$

provided that the constant $A$ is big enough. This proves both lemmas.

We resume the proof of Theorem 3.1 and denote by $\mathcal{H}_{j}$ the linear span in $\mathcal{H}$ of the vectors $\left\{v_{z}+w_{z}: z \in Z_{j}\right\}$ where $w_{z}$ are the vectors from Lemma 3.2 . By construction, the subspaces $\mathcal{H}_{j}$ are mutually orthogonal. Let $P_{\mathcal{H}_{j}}$ be the orthogonal projection to $\mathcal{H}_{j}$ and let $u_{z}=v_{z}-P_{\mathcal{H}_{j}} v_{z}$. Then

$$
\left\|u_{z}\right\| \leqslant\left\|w_{z}\right\| \leqslant e^{-\frac{1}{10} A^{2} \rho_{j}^{2}} .
$$

Now, we choose subspaces $\mathcal{H}_{j}^{*}$ orthogonal to all subspaces $\mathcal{H}_{j}$ and orthogonal to each other, and choose the isometries $S_{j}$ that move all the vectors $u_{z}$ with $z \in Z_{j}$ to $\mathcal{H}_{j}^{*}$. At last, we set

$$
\widetilde{v}_{z}=P_{\mathcal{H}_{j}} v_{z}+S_{j} u_{z},
$$

and introduce the new bunches of unit vectors $\widetilde{N}_{j}=\left\{\widetilde{v}_{z}: z \in Z_{j}\right\}$. The new bunches are mutually orthogonal. Indeed, if $z \in Z_{j}$ and $\zeta \in Z_{k}$ with $k \neq j$, then

$$
\left\langle\widetilde{v}_{z}, \widetilde{v}_{\zeta}\right\rangle=\left\langle P_{\mathcal{H}_{j}} v_{z}+S_{j} u_{z}, P_{\mathcal{H}_{k}} v_{\zeta}+S_{k} u_{\zeta}\right\rangle=0
$$

since the subspaces $\mathcal{H}_{j}, \mathcal{H}_{j}^{*}, \mathcal{H}_{k}, \mathcal{H}_{k}^{*}$ are mutually orthogonal for $k \neq j$. On the other hand, the angles between the vectors within the same bunch remain the same: for $z, \zeta \in Z_{j}$, we have

$$
\left\langle\widetilde{v}_{z}, \widetilde{v}_{\zeta}\right\rangle=\left\langle P_{\mathcal{H}_{j}} v_{z}+S_{j} u_{z}, P_{\mathcal{H}_{j}} v_{\zeta}+S_{j} u_{\zeta}\right\rangle=\left\langle P_{\mathcal{H}_{j}} v_{z}, P_{\mathcal{H}_{j}} v_{\zeta}\right\rangle+\left\langle u_{z}, u_{\zeta}\right\rangle=\left\langle v_{z}, v_{\zeta}\right\rangle .
$$

Hence the vectors $\left\{\widetilde{v}_{z}\right\}$ define the same Gaussian process on $Z_{j}$ as $\left\{v_{z}\right\}$, and the new processes are independent.

We set $M_{j}=e^{\frac{1}{20} A^{2} \rho_{j}^{2}}$ and note that $\left\|\widetilde{v}_{z}-v_{z}\right\| \leqslant 2\left\|u_{z}\right\| \leqslant 2 M_{j}^{-2}$ for all $z \in Z_{j}$. Then

$$
\mathbb{P}\left\{\left|\widetilde{v}_{z}-v_{z}\right|>t\right\}=e^{-\left(t /\left\|\widetilde{v}_{z}-v_{z}\right\|\right)^{2}} \leqslant e^{-\left(t M_{j}^{2} / 2\right)^{2}}, \quad z \in Z_{j} .
$$


Plugging in this estimate with $t=\frac{1}{M_{j}}$, we get

$$
\mathbb{P}\left\{\sup _{z \in Z_{j}}\left|\widetilde{v}_{z}-v_{z}\right|>\frac{1}{M_{j}}\right\} \leqslant \# Z_{j} \cdot e^{-\frac{1}{4} M_{j}^{2}}
$$

Since

we have

$$
\# Z_{j} \leqslant A^{2} \rho_{j}^{2} \cdot \# \mathcal{C}_{j} \lesssim A^{2} \rho_{j}^{2} \cdot\left(1+d\left(K_{j}\right)\right)^{2} \lesssim A^{2} \rho_{j}^{2} \cdot e^{2 \rho_{j}^{2}}
$$

$$
\mathbb{P}\left\{\sup _{z \in Z_{j}}\left|\widetilde{v}_{z}-v_{z}\right|>\frac{1}{M_{j}}\right\} \lesssim A^{2} \rho_{j}^{2} \cdot e^{2 \rho_{j}^{2}} \cdot e^{-\frac{1}{4} M_{j}^{2}}<e^{-c M_{j}^{2}}
$$

provided that $A$ is sufficiently big.

Now, we extend the processes $\left\{\widetilde{v}_{z} e^{\frac{1}{2}|z|^{2}}: z \in Z_{j}\right\}$ to independent G.E.F.'s $F_{j}$. We know that $G_{j}^{*}=F^{*}-F_{j}^{*}$ is small on the net $Z_{j}$ with probability very close to 1:

$$
\mathbb{P}\left\{\sup _{z \in Z_{j}}\left|G_{j}^{*}(z)\right|>\frac{1}{M_{j}}\right\}<e^{-c M_{j}^{2}} .
$$

Discarding the event $\left\{\sup _{z \in Z_{j}}\left|G_{j}^{*}(z)\right|>M_{j}^{-1}\right\}$, we assume that

$$
\sup _{z \in Z_{j}}\left|G_{j}^{*}(z)\right| \leqslant \frac{1}{M_{j}}
$$

and estimate the difference $G_{j}^{*}=F^{*}-F_{j}^{*}$ everywhere on $K_{j}$.

Let $\mathcal{D}$ be one of the unit disks with the center $\kappa \in \mathcal{C}_{j}$. It is convenient to move the point $\kappa$ to the origin using the projective translation

$$
\left(T_{\kappa} f\right)(z)=f(\kappa+z) e^{-z \bar{\kappa}} e^{-\frac{1}{2}|\kappa|^{2}} .
$$

Comparing the covariances, it is easy to check that if $F$ is a G.E.F., then $T_{\kappa} F$ is also a G.E.F. [13, Lemma 2.6]. To simplify our notation, we set $G(z)=\left(T_{\kappa} G_{j}\right)(z)$, and denote by $\Lambda$ the set of equidistant points on the unit circumference such that $\Lambda+\kappa \subset Z_{j}$. Due to our assumption (8), the random analytic function $G$ is very small on $\Lambda$ :

$$
|G(\lambda)|=\left|T_{\kappa}\left(F-F_{j}\right)(\lambda)\right| \leqslant \frac{1}{M_{j}} e^{\frac{1}{2}|\lambda+\kappa|^{2}-\frac{1}{2}|\kappa|^{2}-\operatorname{Re}(\lambda \bar{\kappa})}=\frac{1}{M_{j}} e^{\frac{1}{2}}<\frac{2}{M_{j}}
$$

Since $|G| \leqslant\left|T_{\kappa} F\right|+\left|T_{\kappa} F_{j}\right|$, the function $G$ is not too big everywhere on the disk $\{|z| \leqslant 2\}$ with very high probability:

$$
\mathbb{P}\left\{\max _{\{|z| \leqslant 2\}}|G(z)| \geqslant M_{j}\right\} \leqslant e^{-c M_{j}^{2}} .
$$

This follows from Lemma 6 in 14 applied to the G.E.F.'s $T_{\kappa} F$ and $T_{\kappa} F_{j}$.

Now, we suppose that $|G| \leqslant M_{j}$ everywhere in the disk $\{|z| \leqslant 2\}$. Then estimate (9) yields that $G$ is very small everywhere on the disk $\left\{|z| \leqslant \frac{1}{\sqrt{2}}\right\}$. To see this, first, we approximate $G$ by its Taylor polynomial $P_{N}$ of sufficiently large degree $N$. Let

$$
G(z)=\sum_{n \geqslant 0} g_{n} z^{n} .
$$


By Cauchy's inequalities, for each $n \in \mathbb{Z}_{+},\left|g_{n}\right| \leqslant M_{j} \cdot 2^{-n}$, and

$$
\left|\sum_{n \geqslant N+1} g_{n} z^{n}\right| \leqslant \frac{M_{j}}{2^{N}}
$$

Since we wish the approximation error $\max _{D}\left|G-P_{N}\right|$ to be less than $M_{j}^{-1}$, we need to take $2^{N} \geqslant M_{j}^{2}$, that is, $N \approx 2 \log _{2} M_{j}$. Then $\left|P_{N}\right| \leqslant 2 M_{j}^{-1}$ at about $A^{2} \rho_{j}^{2}=$ $20 \log M_{j}$ equidistant points $\lambda$ on the unit circumference. Since the number of these points is bigger than the degree of $P_{N}$, and since these points are equidistant, we have

$$
g_{n}=\frac{1}{\# \Lambda} \sum_{\lambda \in \Lambda} P_{N}(\lambda) \lambda^{-n}, \quad 0 \leqslant n \leqslant N,
$$

whence, $\left|g_{n}\right| \leqslant 2 M_{j}^{-1}$ for $0 \leqslant n \leqslant N$. Thus,

$$
\left|P_{N}(z)\right|<\frac{2}{M_{j}} \frac{\sqrt{2}}{\sqrt{2}-1}<\frac{8}{M_{j}},
$$

and $|G(z)|<\frac{9}{M_{j}}$ for $|z| \leqslant \frac{1}{\sqrt{2}}$.

It is easy to check that $\left|G_{j}^{*}(z)\right|=|G(z-\kappa)| e^{-\frac{1}{2}|z-\kappa|^{2}}$. Hence, for $z \in D\left(\kappa, \frac{1}{\sqrt{2}}\right)$, we have $\left|G_{j}^{*}(z)\right|<\frac{9}{M}$, provided that $|G(z)|<\frac{9}{M_{j}}$ therein. Since the union of all disks of radius $\frac{1}{\sqrt{2}}$ centered at $\mathcal{C}_{j}$ covers $K_{j}$, we see that

$$
\begin{aligned}
& \mathbb{P}\left\{\max _{K_{j}}\left|G_{j}^{*}\right|>e^{-\rho_{j}^{2}}\right\}< \mathbb{P}\left\{\max _{K_{j}}\left|G_{j}^{*}\right|>9 M_{j}^{-1}\right\} \\
& \stackrel{(7),(10)}{<} C \cdot \# \mathcal{C}_{j} \cdot e^{-c M_{j}^{2}}=C \exp \left[2 \rho_{j}^{2}-c e^{\frac{1}{10} A^{2} \rho_{j}^{2}}\right]<\exp \left[-e^{\rho_{j}^{2}}\right],
\end{aligned}
$$

provided that the constant $A$ is big enough. This completes the proof of the almost independence theorem.

\section{Asymptotic normality. Proof of Theorem 1.2}

Without loss of generality, we assume till the end of the proof that $\alpha<2$. We fix a $C_{0}^{\alpha}$-function $h$ supported by the square $\left[-\frac{1}{2}, \frac{1}{2}\right] \times\left[-\frac{1}{2}, \frac{1}{2}\right]$ and fix a big positive $R$ such that $\sigma(R, h) \gtrsim R^{-\alpha+\varepsilon}$. By $\mathcal{S}$ we denote the square $[-1,1] \times[-1,1]$. As above, we put $\bar{n}(R, h)=n(R, h)-\mathbb{E} n(R, h)$.

4.1. Preliminary smoothing. First, we approximate the function $h$ by a $C_{0^{-}}^{2}$ function $h_{R}$ so that

$$
\begin{gathered}
\left\|h-h_{R}\right\|_{\infty} \lesssim R^{-3} \\
\left\|\nabla h_{R}\right\|_{\infty},\left\|\Delta h_{R}\right\|_{\infty} \lesssim R^{M(\alpha)} \\
\left\|h_{R}\right\|_{C^{\alpha}} \lesssim\|h\|_{C^{\alpha}} .
\end{gathered}
$$

For instance, we may take $h_{R}=h * \varphi_{\varepsilon} * \varphi_{\varepsilon}$, where $\varphi_{\varepsilon}=\frac{1}{\pi \varepsilon^{2}} \mathbb{1}_{\{|x|<\varepsilon\}}$ with $\varepsilon=R^{-3 / \alpha}$. This gives us (12) with $M(\alpha)=\frac{6}{\alpha}$. 
Now, $n(R, h)=n\left(R, h_{R}\right)+n\left(R, h-h_{R}\right)$, and

$$
\mathbb{V}\left(R, h-h_{R}\right) \stackrel{\text { Cor } 2.5}{\lesssim} R^{2}\left\|h-h_{R}\right\|_{L^{2}}^{2} \lesssim R^{-4}=o(1) \mathbb{V}(R, h)
$$

since by (1), $V(R, h)$ cannot decay faster than $R^{-2}$. Hence, when proving asymptotic normality of the linear statistics $n(R, h)$, we may replace it by $n\left(R, h_{R}\right)$. To simplify the notation, we omit the subscript and continue to denote the function $h_{R}$ by $h$.

4.2. Separating low and high frequencies. We fix a radial function $\chi \in C_{0}^{\infty}$ with

$$
|\widehat{\chi}(\lambda)-1|=O\left(|\lambda|^{2}\right), \quad \lambda \rightarrow 0
$$

and decompose the function $h$ into low and high frequency parts as follows

$$
h=h * \chi_{R}+\left(h-h * \chi_{R}\right)=h_{\mathrm{L}}+h_{\mathrm{H}} .
$$

Here, as above, $\chi_{R}=R^{2} \chi(R \cdot)$. We put $g=\Delta h_{\mathrm{L}}$.

Lemma 4.1. We have

$$
\left\|h_{\mathrm{H}}\right\|_{\infty} \lesssim\|h\|_{C^{\alpha}} R^{-\alpha}
$$

and

$$
\|g\|_{\infty} \lesssim\|h\|_{C^{\alpha}} R^{2-\alpha}
$$

Proof of Lemma 4.1: We give the proof only in the case $\alpha>1$, the proof in the other case $\alpha \leqslant 1$ is very similar. Since the function $\chi$ is radial, we have

$h_{\mathrm{H}}(x)=\int_{\mathbb{R}^{2}} h(x+y) \chi_{R}(y) \mathrm{d} A(y)=\int_{\mathbb{R}^{2}}[h(x+y)-h(x)-\langle\nabla h(x), y\rangle] \chi_{R}(y) \mathrm{d} A(y)$

(we've used that $\chi_{R}$ is orthogonal to linear functions). Therefore,

$$
\left\|h_{\mathrm{H}}\right\|_{\infty} \lesssim\|h\|_{C^{\alpha}} \int_{\mathbb{R}^{2}}|y|^{\alpha}\left|\chi_{R}(y)\right| \mathrm{d} A(y) \lesssim\|h\|_{C^{\alpha}} R^{-\alpha}
$$

Next,

$g(x)=\int_{\mathbb{R}^{2}} h(x+y) \Delta \chi_{R}(y) \mathrm{d} A(y)=\int_{\mathbb{R}^{2}}[h(x+y)-h(x)-\langle\nabla h(x), y\rangle] \Delta \chi_{R}(y) \mathrm{d} A(y)$,

whence

$$
\|g\|_{\infty} \lesssim\|h\|_{C^{\alpha}} \int_{\mathbb{R}^{2}}|y|^{\alpha}\left|\Delta \chi_{R}(y)\right| \mathrm{d} A(y) \lesssim\|h\|_{C^{\alpha}} R^{2-\alpha},
$$

completing the proof.

Lemma 4.2. We have

$$
\left\|h_{\mathrm{H}}\right\|_{L^{2}}^{2} \lesssim R^{-2} \mathbb{V}(R, h)
$$

and

$$
\|g\|_{L^{2}}^{2} \lesssim R^{2} \mathbb{V}(R, h)
$$


Proof of Lemma 4.2. We have

$$
R^{2}\left\|h_{\mathrm{H}}\right\|_{L^{2}}^{2}=R^{2} \int_{\mathbb{R}^{2}}|\widehat{h}(\lambda)|^{2} \cdot\left|1-\widehat{\chi}\left(\frac{1}{R} \lambda\right)\right|^{2} \mathrm{~d} A(\lambda) .
$$

Since the Fourier transform of the cut-off function $\chi$ satisfies (14), Theorem 1.1 yields (17). The second estimate (18) follows from Corollary 2.7.

Now, we split the linear statistics $\bar{n}(R, h)$ into low and high frequency parts:

$$
\begin{array}{r}
\bar{n}(R, h)=\frac{1}{2 \pi R^{2}} \int_{R \mathcal{S}} g\left(\frac{x}{R}\right) U(x) \mathrm{d} A(x)+\frac{1}{2 \pi} \int_{R \mathcal{S}} h_{\mathrm{H}}\left(\frac{x}{R}\right) \Delta U(x) \mathrm{d} A(x) \\
=\bar{n}_{\mathrm{L}}(R, h)+\bar{n}_{\mathrm{H}}(R, h) .
\end{array}
$$

Here, as before, $U=\log \left|F^{*}\right|$ is the random potential. Note that $\mathbb{E} \bar{n}_{L}=0$ (since $g=\Delta h_{\mathrm{L}}$, the integral of $g$ against constants vanishes), and therefore $\mathbb{E} \bar{n}_{\mathrm{H}}=0$.

4.3. Squares and corridors. We fix the parameters $0<\beta<\gamma<\frac{1}{2} \varepsilon$ with $\varepsilon$ taken from the assumptions of the theorem, and partition the plane into the squares $\mathcal{Q}$ with sides parallel to the coordinate axes and of length $R^{\gamma-1}$ separated by the corridors of width $R^{\beta-1}$. We denote by $K$ the union of the corridors. The partition is defined up to translations of the position of the system of corridors. We use this freedom to discard the contribution of the corridors. Averaging over translations, it is not difficult to see that there exists a position of the corridors such that

$$
\left\|g \mathbb{1}_{K}\right\|_{L^{2}}^{2} \lesssim R^{-(\gamma-\beta)}\|g\|_{L^{2}}^{2}
$$

and

$$
\left\|h_{\mathrm{H}} \mathbb{1}_{K}\right\|_{L^{2}}^{2} \lesssim R^{-(\gamma-\beta)}\left\|h_{\mathrm{H}}\right\|_{L^{2}}^{2} .
$$

Then we immediately obtain

Lemma 4.3. There exists a position of the corridors such that

$$
\begin{aligned}
& \mathbb{V}\left\{\frac{1}{R^{2}} \int_{R K} g\left(\frac{x}{R}\right) U(x) \mathrm{d} A(x)\right\}+\mathbb{V}\left\{\int_{R K} h_{\mathrm{H}}\left(\frac{x}{R}\right) \Delta U(x) \mathrm{d} A(x)\right\} \\
&=o(1) \mathbb{V}(R, h), \quad R \rightarrow \infty .
\end{aligned}
$$

Proof of Lemma 4.3: We fix position of the corridors to satisfy (19) and (20). It follows from Remark 2.4 that for any test function $g$, the variance of the random variable $\int_{\mathbb{R}^{2}} g(x) U(x) \mathrm{d} A(x)$ is $\lesssim\|g\|_{L^{2}}^{2}$. Scaling by $R$, we see that

$$
\begin{aligned}
\mathbb{V}\left\{\frac{1}{R^{2}} \int_{R K} g\left(\frac{x}{R}\right) U(x) \mathrm{d} A(x)\right\} \lesssim \frac{1}{R^{4}} \cdot R^{2}\left\|g \mathbb{1}_{K}\right\|_{L^{2}}^{2} & \\
& \stackrel{\lesssim}{\lesssim} R^{-2-(\gamma-\beta)}\|g\|_{L^{2}}^{2} \stackrel{(18)}{=} o(1) \mathbb{V}(R, h) .
\end{aligned}
$$


Similarly,

$$
\begin{aligned}
& \mathbb{V}\left\{\int_{R K} h_{\mathrm{H}}\left(\frac{x}{R}\right) \Delta U(x) \mathrm{d} A(x)\right\} \stackrel{\text { Cor } \stackrel{2.6}{\lesssim}}{\lesssim} R^{2}\left\|h_{\mathrm{H}} \mathbb{1}_{K}\right\|_{L^{2}}^{2} \\
& \stackrel{\text { (20) }}{\lesssim} R^{-(\gamma-\beta)} R^{2}\left\|h_{\mathrm{H}}\right\|_{L^{2}}^{2} \stackrel{(17)}{=} o(1) \mathbb{V}(R, h) .
\end{aligned}
$$

This proves the lemma.

4.4. Smoothing the indicator-functions of the squares $\mathcal{Q}$. Later, when we will deal with the random variables

$$
\int_{R \mathcal{Q}} h_{\mathrm{H}}\left(\frac{x}{R}\right) \Delta U(x) \mathrm{d} A(x)
$$

it will be convenient to modify the functions $h_{\mathrm{H}} 1_{\mathcal{Q}}$ smoothing them near the boundaries $\partial \mathcal{Q}$ with the "range of smoothing" about $R^{\beta-1}$. We introduce a "smooth indicator function' $\widetilde{\mathbb{1}}_{\mathcal{Q}}$ so that $0 \leqslant \widetilde{\mathbb{1}}_{\mathcal{Q}} \leqslant 1$,

$$
\widetilde{\mathbb{1}}_{\mathcal{Q}}= \begin{cases}1 & \text { on } \mathcal{Q}, \\ 0 & \text { when } \operatorname{dist}(x, \mathcal{Q}) \geqslant \frac{1}{10} R^{\beta-1},\end{cases}
$$

$\left\|\nabla \widetilde{\mathbb{1}}_{\mathcal{Q}}\right\|_{\infty} \lesssim R^{1-\beta}$, and $\left\|\Delta \widetilde{\mathbb{1}}_{\mathcal{Q}}\right\|_{\infty} \lesssim R^{2(1-\beta)}$. Let $\widetilde{\mathcal{Q}}=\operatorname{supp}\left(\widetilde{\mathbb{1}}_{\mathcal{Q}}\right), h_{\mathrm{H}}^{\mathcal{Q}}=h_{\mathrm{H}} \widetilde{\mathbb{1}}_{\mathcal{Q}}$.

Lemma 4.4. We have

(i) $h_{\mathrm{H}}^{\mathcal{Q}}(x)=h_{\mathrm{H}}(x)$ on $\mathcal{Q}$;

(ii) $\left\|h_{\mathrm{H}}^{\mathcal{Q}}\right\|_{\infty} \lesssim\|h\|_{C^{\alpha}} R^{-\alpha}$;

(iii) $\left\|\Delta h_{\mathrm{H}}^{\mathcal{Q}}\right\|_{\infty} \lesssim C(h) R^{M(\alpha)+2}$;

(iv)

$$
\mathbb{V}\left\{\sum_{\mathcal{Q}} \int_{\mathbb{R}^{2}}\left(h_{\mathrm{H}} \mathbb{1}_{\mathcal{Q}}-h_{\mathrm{H}}^{\mathcal{Q}}\right)\left(\frac{x}{R}\right) \Delta U(x) \mathrm{d} A(x)\right\} \lesssim R^{-(\gamma-\beta)} \mathbb{V}(R, h) .
$$

Proof of Lemma 4.4: The properties (i)-(iii) are straightforward. Property (iv) follows from

$$
\begin{aligned}
& \mathbb{V}\left\{\sum_{\mathcal{Q}} \int_{\mathbb{R}^{2}}\left(h_{\mathrm{H}} \mathbb{1}_{\mathcal{Q}}-h_{\mathrm{H}}^{\mathcal{Q}}\right)\left(\frac{x}{R}\right) \Delta U(x) \mathrm{d} A(x)\right\}
\end{aligned}
$$

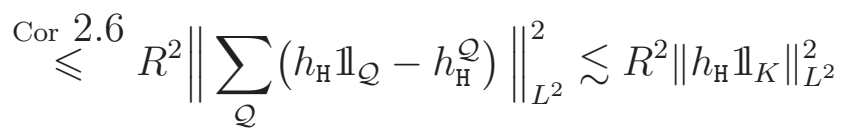

$$
\begin{aligned}
& \stackrel{\text { (15) }}{\lesssim} R^{-(\gamma-\beta)} R^{2}\left\|h_{\mathrm{H}}\right\|_{L^{2}}^{2} \stackrel{\text { (17) }}{\lesssim} R^{-(\gamma-\beta)} \mathbb{V}(R, h) \text {. }
\end{aligned}
$$

This proves the lemma.

Now, we introduce the random variables

$$
\xi_{\mathrm{L}}^{\mathcal{Q}}(R)=\frac{1}{2 \pi R^{2}} \int_{R \mathcal{Q}} g\left(\frac{x}{R}\right) U(x) \mathrm{d} A(x)
$$


and

and set

$$
\xi_{\mathrm{H}}^{\mathcal{Q}}(R)=\frac{1}{2 \pi} \int_{R \mathcal{Q}} h_{\mathrm{H}}^{\mathcal{Q}}\left(\frac{x}{R}\right) \Delta U(x) \mathrm{d} A(x),
$$

$$
\begin{aligned}
\Theta(R) \stackrel{\text { def }}{=} \frac{1}{2 \pi R^{2}} \int_{R K} g\left(\frac{x}{R}\right) U(x) & \mathrm{d} A(x)+\frac{1}{2 \pi} \int_{R K} h_{\mathrm{H}}\left(\frac{x}{R}\right) \Delta U(x) \mathrm{d} A(x) \\
& +\frac{1}{2 \pi} \sum_{\mathcal{Q}} \int_{\mathbb{R}^{2}}\left(h_{\mathrm{H}} \mathbb{1}_{\mathcal{Q}}-h_{\mathrm{H}}^{\mathcal{Q}}\right)\left(\frac{x}{R}\right) \Delta U(x) \mathrm{d} A(x) .
\end{aligned}
$$

Then

$$
\bar{n}(R, h)=\Theta(R)+\sum_{\mathcal{Q}} \underbrace{\left(\xi_{\mathrm{L}}^{\mathcal{Q}}(R)+\xi_{\mathrm{H}}^{\mathcal{Q}}(R)\right)}_{\stackrel{\text { def }}{=} \xi^{\mathcal{Q}}(R)} .
$$

4.5. Probabilistic lemma. To prove the asymptotic normality of the random variables $\bar{n}(R, h)$ we use the following lemma:

Lemma 4.5. Fix $p \geqslant 4$. Assume that $\xi$ is a real random variable with variance $\sigma^{2}$ such that

$$
\xi=\Theta+\sum_{j=1}^{N} \xi_{j}
$$

where

1) $\mathbb{V}\{\Theta\} \leqslant \delta \sigma^{2}$

2) $\sum_{j} \mathbb{E}\left|\xi_{j}\right|^{p} \leqslant \delta \sigma^{p}$;

3) there exist independent random variables $\eta_{j}$ such that

$$
\sum_{j} \mathbb{P}\left\{\left|\xi_{j}-\eta_{j}\right|>\delta N^{-1} \sigma\right\} \leqslant \delta N^{-2}
$$

Then the random variable $\sigma^{-1}(\xi-\mathbb{E} \xi)$ is close in distribution to the normal law when $\delta$ is close to 0 .

Due to the second condition, if $\delta \rightarrow 0$, then automatically $N \rightarrow \infty$.

Proof of Lemma 4.5: using appropriate truncations, we will reduce this lemma to one of the standard versions of the central limit theorem.

Without loss of generality, we assume that $\sigma=1$. Replacing $\delta$ by $2^{p} \delta$, we also assume that $\mathbb{E} \xi=\mathbb{E} \Theta=\mathbb{E} \xi_{j}=0$. Put $\eta_{j}^{\prime}=\operatorname{sgn} \eta_{j} \cdot \min \left\{\left|\eta_{j}\right|, N^{2 / p}\right\}$ and denote

$$
\Omega_{j}=\left\{\left|\xi_{j}-\eta_{j}\right|>\delta N^{-1}\right\}, \quad \Omega_{j}^{\prime}=\left\{\left|\xi_{j}-\eta_{j}^{\prime}\right|>\delta N^{-1}\right\} .
$$

We have

$$
\mathbb{P}\left(\Omega_{j}^{\prime}\right) \leqslant \mathbb{P}\left(\Omega_{j}\right)+\mathbb{P}\left(\left\{\left|\xi_{j}\right|>N^{2 / p}\right\}\right) \leqslant \mathbb{P}\left(\Omega_{j}\right)+N^{-2} \mathbb{E}\left|\xi_{j}\right|^{p},
$$

whence

$$
\sum_{j} \mathbb{P}\left(\Omega_{j}^{\prime}\right) \leqslant \delta N^{-2}+N^{-2} \delta=2 \delta N^{-2}
$$


Next,

$$
\begin{aligned}
\mathbb{E}\left|\xi_{j}-\eta_{j}^{\prime}\right|^{2} & \leqslant \delta^{2} N^{-2}+2 \int_{\Omega_{j}^{\prime}}\left|\xi_{j}\right|^{2} \mathrm{~d} \mathbb{P}+2 \int_{\Omega_{j}^{\prime}}\left|\eta_{j}^{\prime}\right|^{2} \mathrm{~d} \mathbb{P} \\
\leqslant & \leqslant \delta^{2} N^{-2}+2 \int_{\Omega_{j}^{\prime}}\left[N^{-\frac{2}{p}(p-2)}\left|\xi_{j}\right|^{p}+N^{\frac{4}{p}}\right] \mathrm{d} \mathbb{P}+2 \int_{\Omega_{j}^{\prime}} N^{\frac{4}{p}} \mathrm{~d} \mathbb{P} \\
& \leqslant \delta^{2} N^{-2}+2 N^{-1} \mathbb{E}\left|\xi_{j}\right|^{p}+4 N \mathbb{P}\left(\Omega_{j}^{\prime}\right),
\end{aligned}
$$

whence

$$
\sum_{j=1}^{N} \mathbb{E}\left|\xi_{j}-\eta_{j}^{\prime}\right|^{2} \leqslant \delta^{2} N^{-1}+2 N^{-1} \delta+4 N \cdot 2 \delta N^{-2} \leqslant 11 \delta N^{-1}
$$

which together with $\mathbb{E}|\Theta|^{2} \leqslant \delta$ implies that the $L^{2}$-distance between $\xi$ and $\sum_{j} \eta_{j}^{\prime}$ does not exceed $5 \sqrt{\delta}$. In particular, the variance of the sum $\sum_{j} \eta_{j}^{\prime}$ is close to the variance of $\xi$, that is, to 1 .

Now, it remains to show that the independent random variables $\eta_{j}^{\prime}$ satisfy the conditions of Lyapunov's version of the central limit theorem. It remains to check that $\sum_{j} \mathbb{E}\left|\eta_{j}^{\prime}\right|^{p}$ is small when $\delta$ is small. Indeed,

$$
\mathbb{E}\left|\eta_{j}^{\prime}\right|^{p}=\int_{\Omega_{j}^{\prime}}\left|\eta_{j}^{\prime}\right|^{p} \mathrm{~d} \mathbb{P}+\int_{\Omega \backslash \Omega_{j}^{\prime}}\left|\eta_{j}^{\prime}\right|^{p} \mathrm{~d} \mathbb{P}=J_{1}+J_{2} .
$$

We have $J_{1} \leqslant N^{2} \mathbb{P}\left(\Omega_{j}^{\prime}\right)$ because $\left|\eta_{j}^{\prime}\right|^{p} \leqslant N^{2}$. We also have

$$
J_{2} \leqslant \mathbb{E}\left[\left|\xi_{j}\right|+\delta N^{-1}\right]^{p} \leqslant 2^{p}\left(\mathbb{E}\left|\xi_{j}\right|^{p}+\delta^{p} N^{-p}\right) .
$$

Thus, we finally get

$$
\mathbb{E}\left|\eta_{j}^{\prime}\right|^{p} \leqslant N^{2} \mathbb{P}\left(\Omega_{j}^{\prime}\right)+2^{p} \mathbb{E}\left|\xi_{j}\right|^{p}+2^{p} \delta^{p} N^{-p}
$$

and

$$
\sum_{j=1}^{N} \mathbb{E}\left|\eta_{j}^{\prime}\right|^{p} \leqslant N^{2} \cdot 2 \delta N^{-2}+2^{p} \delta+2^{p} \delta^{p} N^{1-p} \leqslant 2\left(2^{p}+1\right) \delta,
$$

finishing the proof.

In our situation, by Lemmas 4.3 and 4.4 , the variance of the random variable $\Theta$ is asymptotically negligible compared to the variance of $\bar{n}(R, h)$ as $R \rightarrow \infty$. This gives us condition 1) in Lemma 4.5.

Now, we check that the sum of the $p$-th moments of the random variables $\xi^{\mathcal{Q}}(R)$ is asymptotically negligible compared to the $\frac{1}{2} p$-th power of the variance of $\bar{n}(R, h)$. This will give us condition 2) in Lemma 4.5. We choose sufficiently large $p=p(\varepsilon)$ (we shall have $p(\varepsilon) \rightarrow \infty$ as $\varepsilon \rightarrow 0$ ). 
4.6. The $p$-th moment estimate. We have

$$
\begin{aligned}
& \mathbb{E}\left|\xi_{\mathrm{L}}^{\mathcal{Q}}\right|^{p}=\mathbb{E}\left|\frac{1}{2 \pi R^{2}} \int_{R \mathcal{Q}} g\left(\frac{x}{R}\right) U(x) \mathrm{d} A(x)\right|^{p} \\
& \leqslant \frac{\|g\|_{\infty}^{p}}{(2 \pi R)^{2 p}} A(R \mathcal{Q})^{p-1} \int_{R \mathcal{Q}} \mathbb{E}|U(x)|^{p} \mathrm{~d} A(x) \\
&=\frac{\|g\|_{\infty}^{p}}{(2 \pi R)^{2 p}} A(R \mathcal{Q})^{p} \mathbb{E}|U(0)|^{p}
\end{aligned}
$$

(in the last line, we used the distribution invariance of the random potential $U$ ). Using estimate (16) and recalling that the area of the square $R \mathcal{Q}$ is $R^{2 \gamma}$, we get

$$
\mathbb{E}\left|\xi_{\mathrm{L}}^{\mathcal{Q}}\right|^{p} \leqslant C^{p}\|h\|_{C^{\alpha}}^{p}\left[\frac{R^{2-\alpha}}{R^{2}} R^{2 \gamma}\right]^{p}=C(p, h) R^{(2 \gamma-\alpha) p}
$$

The $p$-th moment of the high-frequency component $\mathbb{E}\left|\xi_{\mathrm{H}}^{\mathcal{Q}}\right|^{p}$ has the same upper bound. Indeed,

$$
\begin{aligned}
& \mathbb{E}\left|\xi_{\mathrm{H}}^{\mathcal{Q}}\right|^{p}=\mathbb{E} \mid \frac{1}{2 \pi} \int_{\mathbb{R}^{2}} h_{\mathrm{H}}^{\mathcal{Q}}\left.\left(\frac{x}{R}\right) \Delta U(x) \mathrm{d} A(x)\right|^{p} \\
& \leqslant\left\|h_{\mathrm{H}}^{\mathcal{Q}}\right\|_{\infty}^{p} \mathbb{E}\left(n_{F}(R \widetilde{\mathcal{Q}})+A(R \widetilde{\mathcal{Q}})\right)^{p} \\
& \quad \leqslant C(p, h) R^{-p \alpha}\left(\mathbb{E} n_{F}(R \widetilde{\mathcal{Q}})^{p}+A(R \widetilde{\mathcal{Q}})^{p}\right)
\end{aligned}
$$

where, as before, $\widetilde{\mathcal{Q}}$ denotes the support of the function $\widetilde{\mathbb{1}}_{\mathcal{Q}}$. We've used that the positive part of the signed measure $\frac{1}{2 \pi} \Delta U \mathrm{~d} A(x)$ is the counting measure $n_{F}$ of zeroes of $F$ while the negative part is the area measure with the scaling coefficient $\frac{1}{2}$.

By distribution invariance of zeroes, estimating the $p$-th moment $\mathbb{E} n_{F}(R \widetilde{\mathcal{Q}})^{p}$, we may assume that the square $\mathcal{Q}$ is centered at the origin. We take $\rho \simeq R^{\gamma}$ so that $\widetilde{\mathcal{Q}} \subset\{|z| \leqslant \rho\}$ and denote by $n_{F}(\rho)$ the number of zeroes of $F$ in the disk $\{|z| \leqslant \rho\}$. Then $\mathbb{E} n_{F}(R \widetilde{\mathcal{Q}})^{p} \leqslant \mathbb{E} n_{F}(\rho)^{p}$. By Jensen's formula,

$$
n_{F}(\rho) \leqslant \frac{1}{2 \pi} \int_{0}^{2 \pi} \log ^{+}\left|F\left(e \rho e^{\mathrm{i} \theta}\right)\right| \mathrm{d} \theta+\log ^{-}|F(0)|
$$

and

$$
n_{F}(\rho)^{p} \leqslant 2^{p}\left\{\frac{1}{2 \pi} \int_{0}^{2 \pi}\left(\log ^{+}\left|F\left(e \rho e^{\mathrm{i} \theta}\right)\right|\right)^{p} \mathrm{~d} \theta+\left(\log ^{-}|F(0)|\right)^{p}\right\} .
$$

We have

$$
\log ^{+}\left|F\left(e \rho e^{\mathrm{i} \theta}\right)\right| \leqslant \log ^{+}\left|F^{*}\left(e \rho e^{\mathrm{i} \theta}\right)\right|+\frac{1}{2}(e \rho)^{2},
$$

whence,

$$
\left(\log ^{+}\left|F\left(e \rho e^{\mathrm{i} \theta}\right)\right|\right)^{p} \leqslant C(p)\left\{\left(\log ^{+}\left|F^{*}\left(e \rho e^{\mathrm{i} \theta}\right)\right|\right)^{p}+\rho^{2 p}\right\} .
$$

Since the distribution of the normalized function $F^{*}(z)$ does not depend on $z$, and $F^{*}(0)=F(0)$ is a standard complex Gaussian random variable, we have

$$
\mathbb{E}\left\{\left(\log ^{+}\left|F^{*}\left(e \rho e^{\mathrm{i} \theta}\right)\right|\right)^{p}\right\}=\mathbb{E}\left\{\left(\log ^{+}|F(0)|\right)^{p}\right\} .
$$


Therefore, since $\rho>1$,

$$
\mathbb{E} n_{F}(\rho)^{p} \leqslant C(p)\left[\rho^{2 p}+\mathbb{E}|\log | F(0)||\right]^{p} \leqslant C_{1}(p) \rho^{2 p},
$$

and recalling that $\rho \simeq R^{\gamma}$, we finally get

$$
\mathbb{E}\left|\xi_{\mathrm{H}}^{\mathcal{Q}}\right|^{p} \leqslant C(p, h) R^{p(2 \gamma-\alpha)} .
$$

Since the total number of squares $\mathcal{Q}$ is of order $R^{2-2 \gamma}$, we get

$$
\sum_{\mathcal{Q}}\left(\mathbb{E}\left|\xi_{\mathrm{L}}^{\mathcal{Q}}\right|^{p}+\mathbb{E}\left|\xi_{\mathrm{H}}^{\mathcal{Q}}\right|^{p}\right) \leqslant C(p, h) R^{2-2 \gamma} \cdot R^{(2 \gamma-\alpha) p} .
$$

On the other hand, by the assumption of the theorem we are proving,

$$
\sigma(R, h)^{p} \gtrsim c(p, h) R^{(\varepsilon-\alpha) p} .
$$

Since $0<2 \gamma<\varepsilon$, choosing $p$ sufficiently big, we will have

$$
\sum_{\mathcal{Q}} \mathbb{E}\left|\xi^{\mathcal{Q}}\right|^{p}=o(1) \sigma(R, h)^{p}, \quad R \rightarrow \infty .
$$

4.7. Correcting the potential. Now, we combine the low and high frequency terms. We set

$$
g^{\mathcal{Q}}=g \mathbb{1}_{\mathcal{Q}}+\Delta h_{\mathrm{H}}^{\mathcal{Q}},
$$

and note that the functions $g^{\mathcal{Q}}$ have disjoint supports (provided that $R$ is big enough) and that

$$
\left\|g^{\mathcal{Q}}\right\|_{\infty} \leqslant C(h) R^{M(\alpha)+2}
$$

with $M(\alpha)$ taken from (12). Then

$$
\xi^{\mathcal{Q}}(R)=\xi_{\mathrm{L}}^{\mathcal{Q}}(R)+\xi_{\mathrm{H}}^{\mathcal{Q}}(R)=\frac{1}{2 \pi R^{2}} \int_{\mathbb{R}^{2}} g^{\mathcal{Q}}\left(\frac{x}{R}\right) U(x) \mathrm{d} A(x) .
$$

It remains to approximate the random variables $\xi^{\mathcal{Q}}(R)$ by independent ones. This will be done in two steps. First, we correct the random potential $U=\log \left|F^{*}\right|$ replacing the logarithmic kernel by a bounded Lipschitz function $L$ with the Lipschitz norm $R^{\frac{1}{2} B}$.

Given $R \geqslant 2$ and $B \geqslant 1$, let

$$
L(t)=\left\{\begin{aligned}
\log t, & \text { when }|\log t| \leqslant \frac{1}{2} B \log R, \\
\frac{1}{2} B \log R, & \text { when } \log t>\frac{1}{2} B \log R, \\
-\frac{1}{2} B \log R, & \text { when } \log t<-\frac{1}{2} B \log R,
\end{aligned}\right.
$$

let $U_{\text {cor }}=L\left(F^{*}\right)$ be the corrected potential, and let

$$
\xi_{\text {cor }}^{\mathcal{Q}}(R)=\frac{1}{2 \pi R^{2}} \int_{R \mathcal{Q}} g^{\mathcal{Q}}\left(\frac{x}{R}\right) U_{\text {cor }}(x) \mathrm{d} A(x) .
$$

be the corrected random variables.

Lemma 4.6.

$$
\sum_{\mathcal{Q}} \mathbb{E}\left|\xi_{\text {cor }}^{\mathcal{Q}}-\xi^{\mathcal{Q}}\right| \lesssim R^{-12}
$$

provided that $B>M(\alpha)+15$ and that $R$ is big enough. 
Proof of Lemma 4.6. We have

$$
\begin{aligned}
\sum_{\mathcal{Q}}\left|\xi_{\text {cor }}^{\mathcal{Q}}-\xi^{\mathcal{Q}}\right| \leqslant \frac{1}{2 \pi R^{2}} & \int_{\mathbb{R}^{2}}\left(\sum_{\mathcal{Q}}\left|g^{\mathcal{Q}}\right|\right)\left|U_{\text {cor }}(x)-U(x)\right| \mathrm{d} A(x) \\
& \leqslant \frac{1}{2 \pi R^{2}} \sup _{\mathcal{Q}}\left\|g^{\mathcal{Q}}\right\|_{\infty} \int_{\{|x| \leqslant 2 R\}}\left|U_{\text {cor }}(x)-U(x)\right| \mathrm{d} A(x)
\end{aligned}
$$

We've used that the supports of the functions $g^{\mathcal{Q}}$ are disjoint and contained in the disk $\{|x| \leqslant 2 R\}$. The potentials $U_{\text {cor }}(x)$ and $U(x)$ are different only when $\left|F^{*}(x)\right| \leqslant R^{-B / 2}$ or $\left|F^{*}(x)\right| \geqslant R^{B / 2}$. Hence, the right-hand side does not exceed

$$
\begin{aligned}
& \frac{\sup _{\mathcal{Q}}\left\|g^{\mathcal{Q}}\right\|_{\infty}}{2 \pi R^{2}} \int_{\{|x| \leqslant 2 R\}}|U(x)|\left(\mathbb{1}_{\left|F^{*}\right| \leqslant R^{-B / 2}}+\mathbb{1}_{\left|F^{*}\right| \geqslant R^{B / 2}}\right) \mathrm{d} A(x) \\
& \quad \leqslant C(h) R^{M(\alpha)} \int_{\{|x| \leqslant 2 R\}}|U(x)|\left(\mathbb{1}_{\left|F^{*}\right| \leqslant R^{-B / 2}}+\mathbb{1}_{\left|F^{*}\right| \geqslant R^{B / 2}}\right) \mathrm{d} A(x) .
\end{aligned}
$$

Here, we've used that $\left\|g^{\mathcal{Q}}\right\|_{\infty} \leqslant C(h) R^{M(\alpha)+2}$. Therefore,

$$
\begin{aligned}
& \sum_{\mathcal{Q}} \mathbb{E}\left|\xi_{\text {cor }}^{\mathcal{Q}}-\xi^{\mathcal{Q}}\right| \leqslant C(h) R^{M(\alpha)} \\
& \cdot \int_{\{|x| \leqslant 2 R\}} \mathbb{E}\left\{|U(x)|\left(\mathbb{1}_{\left|F^{*}\right| \leqslant R^{-B / 2}}+\mathbb{1}_{\left|F^{*}\right| \geqslant R^{B / 2}}\right)\right\} \mathrm{d} A(x) .
\end{aligned}
$$

Due to the translation invariance of the distribution of $\left|F^{*}\right|$,

$$
\begin{aligned}
\mathbb{E}\left\{|U(x)|\left(\mathbb{1}_{\left|F^{*}\right| \leqslant R^{-B / 2}}+\mathbb{1}_{\left|F^{*}\right|} \geqslant R^{B / 2}\right)\right\} & \\
& =\mathbb{E}\left\{|\log | \zeta|| \mathbb{1}_{|\zeta| \leqslant R^{-B / 2}}\right\}+\mathbb{E}\left\{|\log | \zeta|| \mathbb{1}_{|\zeta| \geqslant R^{B / 2}}\right\}
\end{aligned}
$$

where $\zeta$ is a standard complex Gaussian random variable. The expectations on the right-hand side are readily estimated:

$$
\begin{aligned}
\mathbb{E}\left\{|\log | \zeta|||| \zeta \mid \leqslant R^{-B / 2}\right\} \leqslant \int_{\left\{|\zeta| \leqslant R^{-B / 2}\right\}} & \log \frac{1}{|\zeta|} \mathrm{d} A(\zeta) \\
& \lesssim \int_{0}^{R^{-B / 2}}\left(\log \frac{1}{r}\right) r \mathrm{~d} r \lesssim B R^{-B} \log R
\end{aligned}
$$

and

$$
\begin{aligned}
\mathbb{E}\left\{|\log | \zeta|||| \zeta \mid \geqslant R^{B / 2}\right\} \leqslant \int_{\left\{|\zeta| \geqslant R^{B / 2}\right\}} & (\log |\zeta|) e^{-|\zeta|^{2}} \mathrm{~d} A(\zeta) \\
& \lesssim \int_{R^{B / 2}}^{\infty}(\log r) e^{-r^{2}} r \mathrm{~d} r \lesssim e^{-R^{B}} B \log R .
\end{aligned}
$$

Whence,

$$
\sum_{\mathcal{Q}} \mathbb{E}\left|\xi_{\text {cor }}^{\mathcal{Q}}-\xi^{\mathcal{Q}}\right| \leqslant B C(h) R^{M(\alpha)+2-B} \log R<R^{-12},
$$

provided that $B>M(\alpha)+15$ and that $R$ is big enough. 
4.8. From the almost independent random variables to the independent ones. At last, using Theorem [3.1, we approximate the random variables $\xi_{\text {cor }}^{\mathcal{Q}}$ by the independent ones $\eta^{\mathcal{Q}}$. We apply Theorem 3.1 to the compact sets $K=R \widetilde{\mathcal{Q}}$ and the values $\rho_{\mathcal{Q}}=R^{\frac{1}{2} \beta}$ which are much bigger than $\sqrt{\log (3+d(R \widetilde{\mathcal{Q}}))}$. We assume that $R$ is big enough. Then we get a collection of independent G.E.F.'s $F_{\mathcal{Q}}$ such that for each square $\mathcal{Q}$,

$$
F^{*}=F_{\mathcal{Q}}^{*}+G_{\mathcal{Q}}^{*} \quad \text { on } R \widetilde{\mathcal{Q}},
$$

with

$$
\mathbb{P}\left\{\max _{R \widetilde{\mathcal{Q}}}\left|G_{\mathcal{Q}}^{*}\right| \geqslant e^{-R^{\beta}}\right\} \lesssim \exp \left[-e^{R^{\beta}}\right]
$$

We set

$$
\eta^{\mathcal{Q}}(R)=\frac{1}{2 \pi R^{2}} \int_{\mathbb{R}^{2}} g^{\mathcal{Q}}\left(\frac{x}{R}\right) L\left(\left|F_{\mathcal{Q}}^{*}(x)\right|\right) \mathrm{d} A(x)
$$

and check that

$$
\sum_{\mathcal{Q}} \mathbb{P}\left\{\left|\xi^{\mathcal{Q}}-\eta^{\mathcal{Q}}\right| \geqslant \delta N^{-2} \sigma\right\} \leqslant \delta N^{-2}
$$

with $\delta=\frac{1}{R}, N \approx R^{2-2 \gamma}$, and $\sigma(R, h) \geqslant c(h) R^{-1}$. This will give us condition 3 ) of Lemma 4.5. With these values of the parameters $\delta, N$, and $\sigma$, we have $\delta N^{-2} \sigma>R^{-6}$ and $\delta N^{-2}>R^{-5}$, provided that $R$ is big enough. Hence, it suffices to check that

$$
\sum_{\mathcal{Q}} \mathbb{P}\left\{\left|\xi^{\mathcal{Q}}-\xi_{\text {cor }}^{\mathcal{Q}}\right| \geqslant R^{-6}\right\}<R^{-5}
$$

and that

$$
\sum_{\mathcal{Q}} \mathbb{P}\left\{\left|\xi_{\text {cor }}^{\mathcal{Q}}-\eta^{\mathcal{Q}}\right| \geqslant R^{-6}\right\}<R^{-5} .
$$

The first estimate follows from Lemma 4.6, so we need to check only the second one.

We have

$$
\begin{aligned}
& \left|\xi_{\text {cor }}^{\mathcal{Q}}-\eta^{\mathcal{Q}}\right| \leqslant \frac{1}{2 \pi R^{2}} \int_{\mathbb{R}^{2}}\left|g^{\mathcal{Q}}\left(\frac{x}{R}\right)\right|\left|L\left(F_{\mathcal{Q}}^{*}(x)+G_{\mathcal{Q}}^{*}(x)\right)-L\left(F_{\mathcal{Q}}^{*}(x)\right)\right| \mathrm{d} A(x) \\
& \leqslant \frac{\left\|g^{\mathcal{Q}}\right\|_{\infty}}{R^{2}} \cdot\|L\|_{\text {Lip }} \cdot \max _{R \widetilde{\mathcal{Q}}}\left|G_{\mathcal{Q}}^{*}\right| \cdot A(R \mathcal{Q}) \\
& \lesssim \frac{C(h) R^{M(\alpha)+2}}{R^{2}} \cdot R^{\frac{1}{2} B} \cdot \max _{R \widetilde{\mathcal{Q}}}\left|G_{\mathcal{Q}}^{*}\right| \cdot R^{2-2 \gamma} \\
& \stackrel{B>M(\alpha)+15}{<} R^{2 B} \max _{R \widetilde{\mathcal{Q}}}\left|G_{\mathcal{Q}}^{*}\right|
\end{aligned}
$$


whence,

$$
\begin{array}{rl}
\sum_{\mathcal{Q}} \mathbb{P}\left\{\left|\xi_{\text {cor }}^{\mathcal{Q}}-\eta^{\mathcal{Q}}\right|>R^{-6}\right\} \leqslant \sum_{\mathcal{Q}} & \mathbb{P}\left(R^{2 B} \max _{R \widetilde{\mathcal{Q}}}\left|G_{\mathcal{Q}}^{*}\right|>R^{-B}\right) \\
& \leqslant \sum_{\mathcal{Q}} \mathbb{P}\left(\max _{R \widetilde{\mathcal{Q}}}\left|G_{\mathcal{Q}}^{*}\right|>e^{-R^{\beta}}\right) \lesssim N \exp \left[-e^{R^{\beta}}\right]
\end{array}
$$

which is much less than $R^{-5}$, provided that $R$ is big enough. This finishes off the proof of Theorem 1.2.

\section{Abnormal test-FunCtions}

By $\zeta$ and $\zeta_{j}$ we always denote standard complex Gaussian random variables. Let $b=\mathbb{E} \log |\zeta|$. As above, we denote $\bar{n}(R, h)=n(R, h)-\mathbb{E} n(R, h)$.

5.1. The function $h(x)=\log ^{-}|x|$ is abnormal. We start with a simple example of a function $h$ with abnormal fluctuations of linear statistics. Though this function is unbounded, it may be regarded as a toy model for the main example which we give in the next section.

In view of Jensen's integral formula, this function is customary in the entire functions theory. We have

$$
n(R, h)=\sum_{a \in \mathcal{Z}_{F}} \log ^{+} \frac{R}{|a|}=\frac{1}{2 \pi} \int_{0}^{2 \pi} \log \left|F\left(R e^{\mathrm{i} \theta}\right)\right| \mathrm{d} \theta-\log |F(0)| .
$$

Then

$$
\mathbb{E} n(R, h)=\frac{1}{2 \pi} \int_{0}^{2 \pi} \mathbb{E} \log \left|F\left(R e^{\mathrm{i} \theta}\right)\right| \mathrm{d} \theta-\mathbb{E} \log |F(0)|=\left(\frac{1}{2} R^{2}+b\right)-b=\frac{1}{2} R^{2} .
$$

Letting $F^{*}(z)=F(z) e^{-\frac{1}{2}|z|^{2}}$ and $\ell(\zeta)=\log |\zeta|-b$, we see that

$$
\bar{n}(R, h)=\frac{1}{2 \pi} \int_{0}^{2 \pi} \ell\left(F^{*}\left(R e^{\mathrm{i} \theta}\right)\right) \mathrm{d} \theta-\ell\left(F^{*}(0)\right) .
$$

The variance of the first term on the right-hand side equals

$$
\begin{aligned}
\frac{1}{4 \pi^{2}} \int_{0}^{2 \pi} \int_{0}^{2 \pi} \mathbb{E}\left\{\ell\left(F^{*}\left(R e^{\mathrm{i} \theta_{1}}\right)\right) \ell\left(F^{*}\left(R e^{\mathrm{i} \theta_{2}}\right)\right\} \mathrm{d} \theta_{1} \mathrm{~d} \theta_{2}\right. \\
\stackrel{\text { Lemma }}{=} \frac{2.2}{16 \pi^{2}} \sum_{\alpha \geqslant 1} \frac{1}{\alpha^{2}} \int_{0}^{2 \pi} \int_{0}^{2 \pi} e^{-\alpha R^{2}\left|e^{\mathrm{i} \theta_{1}}-e^{\mathrm{i} \theta_{2}}\right|^{2}} \mathrm{~d} \theta_{1} \mathrm{~d} \theta_{2} \\
\leqslant \frac{1}{8 \pi} \sum_{\alpha \geqslant 1} \frac{1}{\alpha^{2}} \int_{0}^{2 \pi} e^{-\alpha R^{2} \sin ^{2} \theta} \mathrm{d} \theta=O\left(\frac{1}{R}\right), \quad R \rightarrow \infty .
\end{aligned}
$$

Therefore, the first term on the right-hand side of (22) can be disregarded for large $R$ 's. Obviously, the fluctuations of the term $\ell\left(F^{*}(0)\right)$ are not normal, hence, the fluctuations of $n(R, h)$ are not normal as well. 
5.2. Abnormal $C^{\alpha}$ functions with $0<\alpha<1$. By $c_{\alpha}$ we denote various positive constants that depend only on $\alpha$. We fix a radial function $\psi \in C_{0}^{\infty}\left(\mathbb{R}^{2}\right)$ that equals 1 when $|x| \leqslant 1$ and vanishes when $|x| \geqslant 2$, and put $h_{\alpha}(x)=|x|^{\alpha} \psi(x)$. Note that for $|x|<1$, we have $\Delta h_{\alpha}(x)=c_{\alpha}|x|^{\alpha-2}$ with some $c_{\alpha}>0$.

We set $\bar{U}(x)=\ell\left(F^{*}(x)\right)=\log \left|F^{*}(x)\right|-b$, and introduce the random variable

$$
\xi_{R}=\int_{|x| \leqslant R}|x|^{\alpha-2} \bar{U}(x) \mathrm{d} A(x) .
$$

By Remark 2.4, the second moment of the integral $\int_{A \leqslant|x| \leqslant B}|x|^{\alpha-2} \bar{U}(x) \mathrm{d} A(x)$ does not exceed $C \int_{A}^{B} r^{2 \alpha-4} \cdot r \mathrm{~d} r<c_{\alpha} A^{-2(1-\alpha)}$. Therefore, for $R \rightarrow \infty$, the random variable $\xi_{R}$ converges in mean square to the random variable

$$
\xi=\int_{\mathbb{R}^{2}}|x|^{\alpha-2} \bar{U}(x) \mathrm{d} A(x) .
$$

Lemma 5.1. The random variable $\xi$ is not a constant one.

Proof of Lemma 5.1. Suppose that $\xi$ is a constant. Since $\mathbb{E} \xi=0$, this means that $\xi=0$ a.s. . Put

$$
\xi_{R}(y)=\int_{|x| \leqslant R}|y-x|^{\alpha-2} \bar{U}(x) \mathrm{d} A(x) .
$$

By the translation invariance of the distribution of $\bar{U}$, for each $y \in \mathbb{R}^{2}$,

$$
\lim _{R \rightarrow \infty} \mathbb{E}\left\{\xi_{R}(y)^{2}\right\}=0 .
$$

Since the random potential $\bar{U}(x)$ belongs to $L_{\text {loc }}^{2}\left(\mathbb{R}^{2}\right)$, the random functions $\xi_{R}(y)$ also belong to $L_{\text {loc }}^{2}\left(\mathbb{R}^{2}\right)$.

We take a function $\chi \in C_{0}^{\infty}\left(\mathbb{R}^{2}\right)$ with positive Fourier transform, and let $k=$ $|x|^{\alpha-2} * \chi$. Then

$$
\int_{|x| \leqslant R} k(x) \bar{U}(x) \mathrm{d} A(x)=\int_{|y| \leqslant R} \chi(y) \xi_{R}(y) \mathrm{d} A(y) \stackrel{L^{2}}{\rightarrow} 0
$$

in mean square, when $R \rightarrow \infty$. Thus,

$$
\int_{\mathbb{R}^{2}} k(x) \bar{U}(x) \mathrm{d} A(x)=0,
$$

and by the translation invariance,

$$
\int_{\mathbb{R}^{2}} k(y-x) \bar{U}(x) \mathrm{d} A(x) \equiv 0, \quad y \in \mathbb{R}^{2} .
$$

As before, the integrals converge in mean square.

The Fourier transform of the function $|x|^{\alpha-2}$ equals $c(\alpha)|\lambda|^{-\alpha}$ with $c(\alpha)>0$, so that $\widehat{k}(\lambda)>0$. Since $k$ is an $L^{2}\left(\mathbb{R}^{2}\right)$-function with positive Fourier transform, any $L^{2}\left(\mathbb{R}^{2}\right)$-function $h(x)$ can be approximated in the $L^{2}\left(\mathbb{R}^{2}\right)$-norm by finite linear 
combinations of translations $k\left(y_{i}-x\right)$ (this is a classical Wiener's theorem). Then, by Remark 2.4, for any $L^{2}\left(\mathbb{R}^{2}\right)$-function $h$,

$$
\int_{\mathbb{R}^{2}} h(x) \bar{U}(x) \mathrm{d} A(x)=0 .
$$

In turn, this yields the absurd conclusion that $\bar{U}$ is the zero function.

\section{Proposition 5.2.}

(i) The random variables $R^{\alpha} \bar{n}\left(R, h_{\alpha}\right)$ converge in mean square to $c_{\alpha} \xi$ as $R \rightarrow \infty$.

(ii) The random variable $\xi$ is not a Gaussian one.

Hence, $R^{\alpha} \sigma\left(R, h_{\alpha}\right)$ converges to a positive limit, and the fluctuations of the linear statistics $n\left(R, h_{\alpha}\right)$ are not asymptotically normal.

Proof of (i) in Proposition 5.2. Since $\Delta h_{\alpha}$ is an $L^{1}$-function, we have

$$
\bar{n}\left(R, h_{\alpha}\right)=\frac{1}{2 \pi R^{2}} \int_{\mathbb{R}^{2}}\left(\Delta h_{\alpha}\right)\left(\frac{x}{R}\right) \bar{U}(x) \mathrm{d} A(x) .
$$

Therefore,

$$
R^{\alpha} \bar{n}\left(R, h_{\alpha}\right)=c_{\alpha} \xi_{R}+\frac{1}{2 \pi R^{2-\alpha}} \int_{R \leqslant|x| \leqslant 2 R}\left(\Delta h_{\alpha}\right)\left(\frac{x}{R}\right) \bar{U}(x) \mathrm{d} A(x) .
$$

Using Remark 2.4, we see that the variance of the second term on the right-hand side does not exceed

$$
c_{\alpha} R^{2 \alpha-4} \int_{R \leqslant|x| \leqslant 2 R}\left|\Delta h_{\alpha}\left(\frac{x}{R}\right)\right|^{2} \mathrm{~d} A(x) \leqslant c_{\alpha} R^{2 \alpha-2} \rightarrow 0
$$

for $R \rightarrow \infty$. Hence, $R^{\alpha} \bar{n}\left(R, h_{\alpha}\right)$ converges in mean square to $c_{\alpha} \xi$.

In what follows, we use some elementary relations:

Lemma 5.3. For $t>0$, we have

$$
\begin{gathered}
\mathbb{E}\left\{|\zeta|^{t}\right\}=\Gamma\left(\frac{1}{2} t+1\right), \\
\Gamma\left(\frac{1}{2} t+1\right) \leqslant e^{b t+C t^{2}},
\end{gathered}
$$

and

$$
e^{-b t} \mathbb{E}\left\{|\zeta|^{t}\right\} \leqslant e^{C t^{2}},
$$

where $C$ is a sufficiently large positive numerical constant.

Proof of Lemma 5.3: For $t \geqslant 0$, we have

$$
\mathbb{E}\left\{|\zeta|^{t}\right\}=\frac{1}{\pi} \int_{\mathbb{C}}|z|^{t} e^{-|z|^{2}} \mathrm{~d} A(z)=\int_{0}^{\infty} r^{t / 2} e^{-r} \mathrm{~d} r=\Gamma\left(\frac{t}{2}+1\right) .
$$

This gives us (23). Then it is easy to check that for $s \geqslant 0$,

$$
\log \Gamma(1+s) \leqslant \frac{\Gamma^{\prime}(1)}{\Gamma(1)} s+C s^{2}
$$


with a sufficiently big positive numerical constant $C$. It remains to note that

$$
b=\mathbb{E}\{\log |\zeta|\}=\frac{1}{\pi} \int_{\mathbb{C}}(\log |z|) e^{-|z|^{2}} \mathrm{~d} A(z)=\frac{1}{2} \int_{0}^{\infty}(\log s) e^{-s} \mathrm{~d} s=\frac{1}{2} \frac{\Gamma^{\prime}(1)}{\Gamma(1)},
$$

completing the proof of (24).

The idea behind the proof of (ii) in Proposition 5.2 is also simple. At each point $x \in \mathbb{R}^{2}$, the random potential $\bar{U}(x)$ is distributed like $\log |\zeta|-b$, whence by (23) $\log \mathbb{E}\left\{e^{t \bar{U}(x)}\right\} \leqslant C t \log t$ for $t \rightarrow+\infty$. Since the random variable $\xi$ is a weighted average of the random potential $\bar{U}(x)$, we expect that $\mathbb{E}\left\{e^{t \xi}\right\} \leqslant e^{o\left(t^{2}\right)}$ for $t \rightarrow+\infty$, which forbids the random variable $\xi$ to be a Gaussian one. To implement this programme, we need to estimate some Laplace transforms.

Lemma 5.4. Suppose $\varphi$ is a non-negative function on $\mathbb{R}^{2}$. Then

(i)

$$
\mathbb{E} \exp \left[\int_{\mathbb{R}^{2}} \varphi \bar{U}\right] \leqslant e^{-b\|\varphi\|_{1}} \Gamma\left(\frac{1}{2}\|\varphi\|_{1}+1\right)
$$

(ii) for each $L>0$,

$$
\mathbb{E} \exp \left[\int_{\mathbb{R}^{2}} \varphi \bar{U}\right] \leqslant e^{\frac{1}{2} \delta(L)\|\varphi\|_{1}+C L^{2}\|\varphi\|_{2}^{2}},
$$

where $\delta(L)=\sum_{k \in \mathbb{Z}^{2} \backslash\{0\}} e^{-L^{2}|k|^{2} / 2}$.

Proof of (i) in Lemma 5.4. By Jensen's inequality,

$$
\exp \left[\int_{\mathbb{R}^{2}} \varphi \bar{U} \mathrm{~d} A\right] \leqslant \int_{\mathbb{R}^{2}} e^{\|\varphi\|_{1} \bar{U}} \frac{\varphi \mathrm{d} A}{\|\varphi\|_{1}} .
$$

Hence,

$$
\begin{aligned}
\mathbb{E} \exp \left[\int_{\mathbb{R}^{2}} \varphi \bar{U} \mathrm{~d} A\right] \leqslant \int_{\mathbb{R}^{2}} \mathbb{E}\left\{e^{\|\varphi\|_{1}\left(\log \left|F^{*}\right|-b\right)}\right\} \frac{\varphi \mathrm{d} A}{\|\varphi\|_{1}} \\
=e^{-b\|\varphi\|_{1}} \mathbb{E}\left\{|\zeta|^{\|\varphi\|_{1}}\right\}=e^{-b\|\varphi\|_{1}} \Gamma\left(\frac{1}{2}\|\varphi\|_{1}+1\right),
\end{aligned}
$$

proving (i).

The second estimate in Lemma 5.4 is more delicate. It's proof uses two lemmas. The first lemma is classical (see Theorem 26 in [7] where it is attributed to Ingham and Jessen).

Lemma 5.5. Suppose $X$ is a measure space with a probability measure $\mu$, and $f: X \rightarrow \mathbb{R}$ is a random function on $X$. Then

$$
\log \mathbb{E} \exp \left[\int_{X} f \mathrm{~d} \mu\right] \leqslant \int_{X} \log \mathbb{E}\{\exp f\} \mathrm{d} \mu .
$$


Proof of Lemma 5.5: Consider the functional $\theta \mapsto \log \mathbb{E} e^{\theta}$ on real-valued random variables. By Hölder's inequality, it is convex: for $0 \leqslant t \leqslant 1$, we have

$$
\begin{aligned}
\log \mathbb{E}\left\{e^{\left.t \theta_{1}+(1-t) \theta_{2}\right)}\right\} \leqslant \log \left\{\left(\mathbb{E}\left\{e^{\theta_{1}}\right\}\right)^{t} \cdot\left(\mathbb{E}\left\{e^{\theta_{2}}\right\}\right)^{1-t}\right\} & \\
& =t \log \left\{\mathbb{E} e^{\theta_{1}}\right\}+(1-t) \log \mathbb{E}\left\{e^{\theta_{2}}\right\} .
\end{aligned}
$$

Then (26) follows from Jensen's inequality.

Lemma 5.6. Let $\zeta_{i}$ be standard complex Gaussian random variables such that for every $i$, we have

$$
\sum_{j: j \neq i}\left|\mathbb{E}\left\{\zeta_{i} \bar{\zeta}_{j}\right\}\right| \leqslant \delta
$$

Then for all $t_{i} \geqslant 0$, we have

$$
\log \mathbb{E} \prod_{i}\left|\zeta_{i}\right|^{t_{i}} \leqslant \frac{\delta}{2} \sum_{i} t_{i}+b \sum_{i} t_{i}+C \sum_{i} t_{i}^{2} .
$$

Proof of Lemma 5.6: Since the matrix with the entries

$$
a_{i j}= \begin{cases}\delta, & i=j \\ -\mathbb{E}\left\{\zeta_{i} \bar{\zeta}_{j}\right\}, & i \neq j\end{cases}
$$

is non-negative definite, we can find complex Gaussian random variables $\eta_{i}$ independent of all $\zeta_{i}$ such that $\mathbb{E}\left\{\eta_{i} \bar{\eta}_{j}\right\}=a_{i j}$. For $z \in \mathbb{C}$, put $\lambda_{i}(z)=\zeta_{i}+z \eta_{i}$. When $z \in \mathbb{T}$, the random variables $\frac{\lambda_{i}(z)}{\sqrt{1+\delta}}$ are independent standard complex Gaussians, therefore

$$
\mathbb{E} \prod_{i}\left|\lambda_{i}(z)\right|^{t_{i}}=\prod_{i} \mathbb{E}\left|\lambda_{i}(z)\right|^{t_{i}}
$$

By Lemma 5.3,

$$
\mathbb{E}\left|\lambda_{i}(z)\right|^{t_{i}} \leqslant(1+\delta)^{\frac{1}{2} t_{i}} e^{b t_{i}+C t_{i}^{2}}
$$

Thus

$$
\log \mathbb{E} \prod_{i}\left|\lambda_{i}(z)\right|^{t_{i}} \leqslant \frac{\delta}{2} \sum_{i} t_{i}+b \sum_{i} t_{i}+C \sum_{i} t_{i}^{2} .
$$

On the other hand, $\prod_{i}\left|\lambda_{i}(z)\right|^{t_{i}}$ is a subharmonic function of $z$, so

$$
\prod_{i}\left|\zeta_{i}\right|^{t_{i}}=\prod_{i}\left|\lambda_{i}(0)\right|^{t_{i}} \leqslant \int_{\mathbb{T}} \prod_{i}\left|\lambda_{i}(z)\right|^{t_{i}} d m(z)
$$

where $m$ is the Haar measure on $\mathbb{T}$. Taking the expectation of both sides, we complete the proof.

Now we can complete the proof of Lemma 5.4 
Proof of (ii) in Lemma 5.4: We have

$$
\begin{aligned}
& \mathbb{E} \exp \left[\int_{\mathbb{R}^{2}} \varphi \bar{U}\right]= \mathbb{E} \exp \left[\frac{1}{L^{2}} \int_{[0, L]^{2}} L^{2} \sum_{k \in \mathbb{Z}^{2}} \varphi(x+k L) \bar{U}(x+k L)\right] \\
& \stackrel{(26)}{\leqslant} \exp \left[\frac{1}{L^{2}} \int_{[0, L]^{2}} \log \mathbb{E} e^{\left.L^{2} \sum_{k} \varphi(x+k L) \bar{U}(x+k L)\right]}\right. \\
&=e^{-b\|\varphi\|_{1}} \exp \left[\frac{1}{L^{2}} \int_{[0, L]^{2}} \log \mathbb{E} \prod_{k \in \mathbb{Z}^{2}}\left|F^{*}(x+k L)\right|^{L^{2} \varphi(x+k L)}\right]
\end{aligned}
$$

To estimate the expectation of the product on the right-hand side, we apply Lemma 5.6. We have

$$
\sum_{k^{\prime}: k^{\prime} \neq k}\left|\mathbb{E}\left\{F^{*}(x+k L) \overline{F^{*}\left(x+k^{\prime} L\right)}\right\}\right|=\sum_{k^{\prime}: k^{\prime} \neq k} e^{-L^{2}\left|k^{\prime}-k\right|^{2} / 2}=\sum_{k \in \mathbb{Z}^{2} \backslash\{0\}} e^{-L^{2}|k|^{2} / 2} .
$$

Denoting the sum on the right-hand side by $\delta(L)$, and applying Lemma 5.6, we get

$$
\begin{aligned}
\log \mathbb{E} & \prod_{k \in \mathbb{Z}^{2}}\left|F^{*}(x+k L)\right|^{L^{2} \varphi(x+k L)} \\
& \leqslant \frac{1}{2} \delta(L) L^{2} \sum_{k \in \mathbb{Z}^{2}} \varphi(x+k L)+C L^{4} \sum_{k \in \mathbb{Z}^{2}} \varphi^{2}(x+k L)+b L^{2} \sum_{k \in \mathbb{Z}^{2}} \varphi(x+k L),
\end{aligned}
$$

whence

$$
\begin{aligned}
& \log \mathbb{E} \exp \left[\int_{\mathbb{R}^{2}} \varphi \bar{U}\right] \leqslant-b\|\varphi\|_{1} \\
&+\frac{1}{L^{2}} \int_{[0, L]^{2}}\left(\frac{1}{2} \delta(L) L^{2} \sum_{k \in \mathbb{Z}^{2}} \varphi(x+k L)+C L^{4} \sum_{k \in \mathbb{Z}^{2}} \varphi^{2}(x+k L)+b L^{2} \sum_{k \in \mathbb{Z}^{2}} \varphi(x+k L)\right) \mathrm{d} x \\
&=\frac{1}{2} \delta(L)\|\varphi\|_{1}+C L^{2}\|\varphi\|_{2}^{2},
\end{aligned}
$$

completing the proof of Lemma 5.6 .

To prove that the random variable $\xi$ is not Gaussian, we use a simple probabilistic lemma:

Lemma 5.7. Let $\varepsilon_{j}>0$ satisfy $S=\sum_{j=0}^{\infty} \varepsilon_{j}<+\infty$. Suppose that real random variables $\xi_{j}$ satisfy

$$
\log \mathbb{E} e^{t \xi_{j}} \leqslant o\left(t^{2}\right) \quad \text { for all } j \geqslant 0, \text { and } t \rightarrow \infty,
$$

and

$$
\log \mathbb{E} e^{t \xi_{j}} \leqslant \varepsilon_{j}^{2} t^{2} \quad \text { for all } j \geqslant 1, \text { and all } t \geqslant 1 \text {. }
$$

Then

$$
\log \mathbb{E} e^{t \sum_{j \geqslant 0} \xi_{j}}=o\left(t^{2}\right) \quad \text { as } t \rightarrow+\infty .
$$

In particular, this implies that $\sum_{j \geqslant 0} \xi_{j}$ is not normal. 
Proof of Lemma 5.7: Let $\delta_{j}=\varepsilon_{j} / S$. By Hölder's inequality, we have

$$
\mathbb{E} e^{t \sum_{j \geqslant 0} \xi_{j}} \leqslant \prod_{j \geqslant 0}\left\{\mathbb{E} e^{\delta_{j}^{-1} t \xi_{j}}\right\}^{\delta_{j}}=\prod_{0 \leqslant j \leqslant N} \cdot \prod_{j>N} \leqslant e^{o\left(t^{2}\right)} \cdot e^{t^{2} S \sum_{j>N} \varepsilon_{j}} .
$$

It remains to note that $\sum_{j>N} \varepsilon_{j} \rightarrow 0$ as $N \rightarrow+\infty$.

At last, everything is ready to show that the random variable $\xi$ is not a Gaussian one, and thus to complete the proof of Proposition 5.2.

Proof of (ii) in Proposition 5.2: We split the integral $\xi$ into the sum of the integrals $\xi_{0}=\int_{|x| \leqslant 1}|x|^{\alpha-2} \bar{U}(x) \mathrm{d} A(x), \quad$ and $\quad \xi_{j}=\int_{2^{j-1}<|x| \leqslant 2^{j}}|x|^{\alpha-2} \bar{U}(x) \mathrm{d} A(x)$ for $j \geqslant 1$.

To show that $\xi$ is not a Gaussian random variable, we check that $\xi_{j}$ 's satisfy assumptions (28) and (29) of Lemma 5.7. By estimate (i) in Lemma 5.4, condition (28) holds for the random variables $\xi_{j}, j \geqslant 0$, even with $O(t \log t)$ on the right-hand side.

To check condition (29) for $\xi_{j}$ 's with $j \geqslant 1$, we apply estimate (ii) in Lemma 5.4 with $\varphi_{j}(x)=t|x|^{\alpha-2} \mathbb{1}_{\left\{2^{j-1}<|x| \leqslant 2^{j}\right\}}(x)$. Note that

$$
\left\|\varphi_{j}\right\|_{1} \leqslant C_{\alpha} t 2^{\alpha j} \text { and }\left\|\varphi_{j}\right\|_{2}^{2} \leqslant C_{\alpha} t^{2} 2^{-2(1-\alpha) j} .
$$

Also note that for $L \geqslant 1, \delta(L)=\sum_{k \in \mathbb{Z}^{2} \backslash\{0\}} e^{-L^{2}|k|^{2} / 2} \leqslant C e^{-c L^{2}}$ with positive numerical constants $c$ and $C$. Thus, estimate (ii) in Lemma 5.4 gives us the upper bound

$$
\log \mathbb{E} e^{t \xi_{j}} \leqslant C_{\alpha} t^{2}\left(e^{-c L^{2}} 2^{\alpha j}+L^{2} 2^{-2(1-\alpha) j}\right)
$$

valid for $t \geqslant 1$ and $L \geqslant 1$. Taking there $L=j$, we see that $\log \mathbb{E} e^{t \xi_{j}} \leqslant C_{\gamma} t^{2} 2^{-2 \gamma j}$ for $t \geqslant 1$ with any $0<\gamma<1-\alpha$. Thus, condition (29) is satisfied with $\varepsilon_{j}=C_{\gamma} 2^{-\gamma j}$, and the conclusion follows.

\section{REFERENCES}

[1] S. Bernstein, Sur l'extension du théoréme limite du calcul des probabilités aux sommes de quantités dépendantes. Math. Ann. 97 (1927), 1-59.

[2] P. Bleher, B. Shiffman, S. Zelditch Universality and scaling of correlations between zeros on complex manifolds, Invent. Math. 142 (2000), 351-395. arXiv: math-ph/9904020

[3] E. Bogomolny, O. Bohigas, P. Leboeuf, Distribution of roots of random polynomials. Phys. Rev. Lett. 68 (1992), 2726-2729; Quantum chaotic dynamics and random polynomials. J. Statist. Phys. 85 (1996), 639-679. arXiv: chao-dyn/9604001

[4] P. J. Forrester and G. Honner, Exact statistical properties of the zeros of complex random polynomials. J. Phys. A 32 (1999), 2961-2981. arXiv: cond-mat/9811142

[5] S. Janson, Gaussian Hilbert Spaces, Cambridge University Press, 1997.

[6] J. H. Hannay, Chaotic analytic zero points: exact statistics for those of a random spin state. J. Phys. A 29 (1996), L101-L105; The chaotic analytic function. J. Phys. A 31 (1998), L755-L761.

[7] G. H. Hardy, J. E. Littlewood, G. Pólya, Inequalities, Cambridge University Press, 1934.

[8] B. Hough, M. Krishnapur, Y. Peres, B. Virág, Zeros of Gaussian Analytic Functions and Determinantal Point Process, Amer. Math. Soc., 2010. Electronic version available at

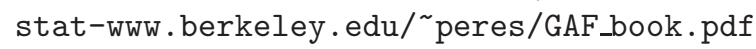


[9] E. Kostlan, Random polynomials and the statistical fundamental theorem of algebra, Preprint, 1987. Available at www.developmentserver.com/ randompolynomials/

[10] M. Krishnapur, Zeros of Random Analytic Functions, Ph.D. Thesis, Berkeley, 2006. arXiv:math/0607504v1

[11] F. Nazarov And M. Sodin, Clustering of correlation functions for random complex zeroes, in preparation.

[12] F. Nazarov and M. Sodin, What is a ... Gaussian entire function? Notices of Amer. Math. Soc., March 2010.

[13] F. Nazarov, M. Sodin, A. Volberg, Transportation to random zeroes by the gradient flow, Geom. and Funct. Anal. 17 (2007), 887-935. arXiv: math.CV/0510654

[14] F. Nazarov, M. Sodin, A. Volberg, The Jancovici - Lebowitz - Manificat law for large fluctuations of random complex zeroes, Comm. Math. Phys. 284 (2008), 833-865. arXiv:0707.3863

[15] B. Rider And B. Virág, The noise in the circular law and the Gaussian free field, Int. Math. Res. Not. IMRN, no 2 (2007), arXiv:math/0606663 Complex determinantal processes and $H^{1}$ noise, Electron. J. Probab. 12 (2007), 1238-1257, arXiv:math/0608785

[16] B. Shiffman And S. Zelditch, Number variance of random zeros on complex manifold. Geom. and Funct. Anal., to appear. arXiv: math.CV/0608743

[17] M. Sodin, Zeros of Gaussian Analytic Functions, Math. Res. Lett. 7 (2000), 371-381. arXiv:math/0007030

[18] M. Sodin AND B. Tsirelson, Random complex zeroes. I. Asympotic normality, Israel J. Math. 144 (2004), 125-149; II. Perturbed Lattice, ibid 152 (2006), 105-124; III. Decay of the hole probability, ibid 147 (2005), 371-379. arxiv: math.CV/0210090, math.CV/0309449, and math.CV/0312258

[19] A. Soshnikov, Gaussian Limit for Determinantal Random Point Fields. Annals of Probab. 30 (2002), 171-187. arXiv:math/0006037

[20] B. Tsirelson, Moderate deviations for random fields and random complex zeroes, arXiv:0801.1050v1.

F.N.: Mathematics Department, University of Wisconsin-Madison, 480 Lincoln Dr., MADISON WI 53706, USA

E-mail address: nazarov@math.wisc.edu

M.S.: School of Mathematical Sciences, Tel Aviv University, Tel Aviv 69978, ISRAEL

E-mail address: sodin@post.tau.ac.il 